\title{
Syntomic cohomology and $p$-adic motivic cohomology
}

\author{
Veronika Ertl and Wiesława Nizioł
}

\begin{abstract}
We prove a mixed characteristic analog of the Beilinson-Lichtenbaum conjecture for $p$-adic motivic cohomology. It gives a description, in the stable range, of $p$-adic motivic cohomology (defined using algebraic cycles) in terms of differential forms. This generalizes a result of Geisser from small Tate twists to all twists. We use as a critical new ingredient the comparison theorem between syntomic complexes and $p$-adic nearby cycles proved recently by Colmez and Nizioł.
\end{abstract}

\section{Introduction}

For a smooth variety over a field of characteristic 0, the Beilinson-Lichtenbaum conjecture states that in a certain stable range, the $p$-adic motivic cohomology is equal to the étale cohomology:

$$
H_{\mathrm{M}}^{i}\left(X, \mathbf{Z} / p^{n}(r)\right) \stackrel{\sim}{\rightarrow} H_{\text {ét }}^{i}\left(X, \mathbf{Z} / p^{n}(r)\right), \quad i \leqslant r .
$$

Here motivic cohomology is defined as the hypercohomology of Bloch's cycle complex $\mathbf{Z} / p^{n}(r)_{\mathrm{M}}$. This conjecture follows (see [SV00]) from the Bloch-Kato conjecture that was proved by Voevodsky and Rost [Wei09].

For a smooth variety over a field of positive characteristic $p$, the analog of the BeilinsonLichtenbaum conjecture states that, in the same stable range, the $p$-adic motivic cohomology is equal to the logarithmic de Rham-Witt cohomology:

$$
H_{\mathrm{M}}^{i}\left(X, \mathbf{Z} / p^{n}(r)\right) \stackrel{\sim}{\rightarrow} H_{\text {ét }}^{i-r}\left(X, W_{n} \Omega_{X, \log }^{r}\right) .
$$

This was proved by Geisser and Levine [GL00].

The purpose of this note is to prove a mixed characteristic analog of the Beilinson-Lichtenbaum conjecture for $p$-adic motivic cohomology. Let $\mathcal{O}_{K}$ be a complete discrete valuation ring with fraction field $K$ of characteristic 0 and with perfect residue field $k$ of characteristic $p$. We fix a uniformizer $\varpi$ of $K$. Let $F$ be the fraction field of the ring of Witt vectors $W(k)$. Let $X$ be a semistable scheme over $\mathcal{O}_{K}$. A scheme $X$ over $\mathcal{O}_{K}$ is called semistable if it is surjective on Spec $\mathcal{O}_{K}$, regular, and there is a distinguished divisor "at infinity" $D_{\infty}$ which is a strict relative normal crossing divisor and which together with the special fiber forms a strict normal crossing divisor. Unless otherwise specified, we will treat $X$ as a log-scheme with the log-structure defined by the special fiber and the divisor at infinity. We assume that the special fiber $X_{0}$ of $X$ is smooth. We show that in the same stable range as above, the $p$-adic motivic cohomology of $X_{\mathrm{tr}}$ - the open

Received 16 April 2016, accepted in final form 27 September 2017.

2010 Mathematics Subject Classification 14F42, 14F20, 11G25.

Keywords: motivic cohomology, syntomic cohomology, $p$-adic nearby cycles.

This journal is (c) Foundation Compositio Mathematica 2019. This article is distributed with Open Access under the terms of the Creative Commons Attribution Non-Commercial License, which permits non-commercial reuse, distribution, and reproduction in any medium, provided that the original work is properly cited. For commercial re-use, please contact the Foundation Compositio Mathematica.

The second author's research was supported in part by the grant ANR-14-CE25. 


\section{Syntomic COHOMOLOGY AND p-ADIC MotiviC COHOMOLOGY}

set where the log-structure is trivial - is equal to the (logarithmic) syntomic-étale cohomology of $X$. This relates algebraic cycles to differential forms.

For a smooth scheme $Y$, we set $H_{\mathrm{M}}^{*}\left(Y, \mathbf{Q}_{p}(r)\right):=H^{*} \operatorname{holim}_{n} \mathrm{R} \Gamma\left(Y_{\mathrm{Zar}}, \mathbf{Z} / p^{n}(r)_{M}\right) \otimes \mathbf{Q}$.

COROLlary 1.1 (Corollary to Theorem 1.2 below). We have the natural isomorphism

$$
H_{\mathrm{M}}^{i}\left(X_{\mathrm{tr}}, \mathbf{Q}_{p}(r)\right) \stackrel{\sim}{\rightarrow} H_{\text {ét }}^{i}(X, \mathcal{E}(r))_{\mathbf{Q}}, \quad i \leqslant r,
$$

where $\mathcal{E}(\cdot)$ denotes the syntomic-étale cohomology complex. If $X$ is proper, this yields the natural isomorphism

$$
H_{\mathrm{M}}^{i}\left(X_{\mathrm{tr}}, \mathbf{Q}_{p}(r)\right) \stackrel{\sim}{\rightarrow} H_{\text {ét }}^{i}(X, \mathcal{S}(r))_{\mathbf{Q}}, \quad i \leqslant r,
$$

where $\mathcal{S}(\cdot)$ denotes the syntomic cohomology complex.

The rational syntomic cohomology $H_{\text {ét }}^{*}(X, \mathcal{S}(r))_{\mathbf{Q}}$ above is that defined in [FM87] as the filtered Frobenius eigenspace of crystalline cohomology. It differs from the one defined in [NN16] by the absence of a log-structure associated to the special fiber. We show in the appendix that it is isomorphic to the logarithmic version of the convergent syntomic cohomology defined in [Niz01], as well as to the rigid syntomic cohomology defined in [Bes00, Gro94].

Corollary 1.1 is a simple consequence of the following theorem, which is the main result of this paper. See [CN17, Section 2.1.1] for what it means for a field to contain enough roots of unity. The field $F$ contains enough roots of unity, and for any $K$, the field $K\left(\zeta_{p^{n}}\right)$, for $n \geqslant c(K)+3$, where $c(K)$ is the conductor of $K$, contains enough roots of unity.

Theorem 1.2. Let $r \geqslant 0$. Let $j_{*}^{\prime}: X_{\mathrm{tr}} \rightarrow X$ be the natural open immersion. If $K$ has enough roots of unity, respectively $K$ does not have enough roots of unity, then there exists a constant $N=N(p, d)$, respectively $N=N(p, d, e)$, depending only on $p$ and the dimension $d$ of $X$, respectively only on $p, d$, and the absolute ramification index $e$ of $K$, such that for $m \geqslant N$, there are natural compatible cycle class maps between complexes of sheaves on the Nisnevich site of $X$ and $X_{0}$, respectively,

$$
\mathrm{cl}_{r}^{\mathrm{syn}}: \mathrm{R} j_{*}^{\prime} \mathbf{Z} / p^{n}(r)_{\mathrm{M}} \rightarrow \mathcal{E}_{n}^{\prime}(r)_{\mathrm{Nis}}, \quad \mathrm{cl}_{r}^{\mathrm{syn}}: i^{*} \mathrm{R} j_{*}^{\prime} \mathbf{Z} / p^{n}(r)_{\mathrm{M}} \rightarrow \mathcal{S}_{n}^{\prime}(r)_{\mathrm{Nis}},
$$

where $i: X_{0} \hookrightarrow X$ is the special fiber of $X$. They are compatible with the étale cycle class maps and are $p^{N r}$-quasi-isomorphisms; that is, the kernels and cokernels of the maps induced on the cohomology sheaves are annihilated by $p^{N r}$.

See Theorem 3.15 for a precise statement concerning the compatibility with the étale cycle class maps.

The syntomic-étale cohomology $\mathcal{E}_{n}^{\prime}(r)$ was defined by Fontaine-Messing [FM87] by gluing syntomic cohomology $\mathcal{S}_{n}^{\prime}(r)$ on $X_{0}$ with étale cohomology on the generic fiber via the relative fundamental exact sequence of $p$-adic Hodge theory. It is a complex of sheaves on the étale site of $X$. We extend this definition to logarithmic schemes (where one replaces syntomic cohomology with logarithmic syntomic cohomology). The Nisnevich version that appears in the above theorem is defined by projecting to the Nisnevich site and truncating at $r$ :

$$
\mathcal{E}_{n}^{\prime}(r)_{\mathrm{Nis}}:=\tau_{\leqslant r} \mathrm{R} \varepsilon_{*} \mathcal{E}_{n}^{\prime}(r), \quad \mathcal{S}_{n}^{\prime}(r)_{\mathrm{Nis}}:=\tau_{\leqslant r} \mathrm{R} \varepsilon_{*} \mathcal{S}_{n}^{\prime}(r),
$$

where $\varepsilon: X_{\text {ét }} \rightarrow X_{\text {Nis }}$ is the natural projection.

The syntomic part of Theorem 1.2 (hence of Corollary 1.1 as well) for twists with $r \leqslant p-2$ (where no constants are needed) was proved by Geisser [Gei04, Theorem 1.3] (his result was conditional on the Bloch-Kato conjecture, which at the time of the publication of his paper was 


\section{ERTL AND W. Nizio€}

not a theorem yet). The key ingredient in his proof is the exact sequence of Kurihara [Kur87] that links syntomic cohomology with $p$-adic nearby cycles coupled with the Beilinson-Lichtenbaum conjecture over fields of characteristic 0 and $p$. Our proof of Theorem 1.2 proceeds in a similar manner using as the main new ingredient the relation between syntomic complexes and $p$-adic nearby cycles proved recently in [CN17].

We will now describe the proof briefly in the case when there is no horizontal log-structure. First, we show that we have the $p^{N r}$-distinguished triangle (on the étale site of $X_{0}$ ), for a constant $N$ as in the theorem,

$$
\mathcal{E}_{n}^{\prime}(r)_{X} \rightarrow \mathcal{E}_{n}^{\prime}(r)_{X \times} \rightarrow W_{n} \Omega_{X_{0}, \log }^{r-1}[-r]
$$

where $W_{n} \Omega_{X_{0}, \log }^{r-1}[-r]$ denotes the logarithmic de Rham-Witt sheaf and $X^{\times}$denotes the scheme $X$ with added log-structure coming from the special fiber. The syntomic-étale cohomology $\mathcal{E}_{n}^{\prime}(r)_{X^{\times}}$ comes equipped with a period map

$$
\alpha_{r}: \mathcal{E}_{n}^{\prime}(r)_{X \times} \rightarrow \mathrm{R} j_{*} \mathbf{Z} / p^{n}(r)_{X_{K}}^{\prime},
$$

where $j_{*}: X_{K} \hookrightarrow X$ and $\mathbf{Z} / p^{n}(r)^{\prime}=\left(p^{a} a !\right)^{-1} \mathbf{Z} / p^{n}(r)$ if $r=(p-1) a+b$ with $a, b \in \mathbf{Z}$ and $0 \leqslant b<p-1$. Projecting it to the Nisnevich site and truncating at $r$, we obtain the Nisnevich syntomic-étale period map

$$
\alpha_{r}: \mathcal{E}_{n}^{\prime}(r)_{X^{\times}, \mathrm{Nis}} \rightarrow \tau_{\leqslant r} \mathrm{R} \varepsilon_{*} \mathrm{R} j_{*} \mathbf{Z} / p^{n}(r)_{X_{K}}^{\prime} .
$$

The computations of $p$-adic nearby cycles via syntomic cohomology from [CN17] imply that this is a $p^{N r}$-quasi-isomorphism, for a constant $N$ as in the theorem. Hence, from (1.1), we obtain the $p^{N r}$-distinguished triangle

$$
\mathcal{E}_{n}^{\prime}(r)_{X, \mathrm{Nis}} \stackrel{\alpha_{r}}{\longrightarrow} \tau_{\leqslant r} \mathrm{R} j_{*} \tau_{\leqslant r} \mathrm{R} \varepsilon_{*} \mathbf{Z} / p^{n}(r)_{X_{K}}^{\prime} \rightarrow i_{*} W_{n} \Omega_{X_{0}, \log }^{r-1}[-r] .
$$

Next, we note that the localization sequence in motivic cohomology yields the following distinguished triangle (on the Nisnevich site of $X$ ):

$$
\mathbf{Z} / p^{n}(r)_{\mathrm{M}} \rightarrow j_{*} \mathbf{Z} / p^{n}(r)_{\mathrm{M}} \rightarrow i_{*} \mathbf{Z} / p^{n}(r-1)_{\mathrm{M}}[-1] .
$$

By the Beilinson-Lichtenbaum conjecture and the computations of Geisser-Levine [GL00] of motivic cohomology in characteristic $p$, we have the cycle class map quasi-isomorphisms

$$
\mathbf{Z} / p^{n}(r)_{\mathrm{M}} \stackrel{\sim}{\rightarrow} \tau_{\leqslant r} \mathrm{R} \varepsilon_{*} \mathbf{Z} / p^{n}(r)_{X_{K}}, \quad \mathbf{Z} / p^{n}(r)_{\mathrm{M}} \stackrel{\sim}{\rightarrow} W_{n} \Omega_{X_{0}, \log }^{r}[-r]
$$

The above triangle becomes

$$
\mathbf{Z} / p^{n}(r)_{\mathrm{M}} \rightarrow j_{*} \tau_{\leqslant r} \mathrm{R} \varepsilon_{*} \mathbf{Z} / p^{n}(r)_{X_{K}} \rightarrow i_{*} W_{n} \Omega_{X_{0}, \log }^{r-1}[-r] .
$$

Since $j_{*} \mathbf{Z} / p^{n}(r)_{\mathrm{M}} \stackrel{\sim}{\rightarrow} \mathrm{R} j_{*} \mathbf{Z} / p^{n}(r)_{\mathrm{M}}$ and $\tau_{\leqslant r} \mathbf{Z} / p^{n}(r)_{\mathrm{M}} \stackrel{\sim}{\rightarrow} \mathbf{Z} / p^{n}(r)_{\mathrm{M}}$, the cycle class map of Theorem 1.2 can now be obtained by comparing sequences (1.2) and (1.3).

Notation and conventions. We assume all the schemes to be locally noetherian. We work in the category of fine log-schemes.

We will use a shorthand for certain homotopy limits. Namely, if $f: C \rightarrow C^{\prime}$ is a map in the differential graded $(\mathrm{dg})$ derived category of abelian groups, we set

$$
\left[C \stackrel{f}{\longrightarrow} C^{\prime}\right]:=\operatorname{holim}\left(C \rightarrow C^{\prime} \leftarrow 0\right)
$$




\section{Syntomic COHOMOLOGY AND $p$-ADiC MOTIVIC COHOMOLOGY}

We set

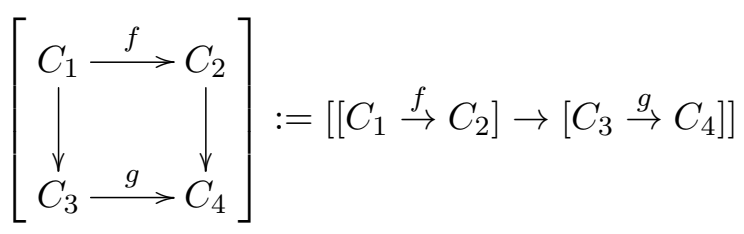

for a commutative diagram (the one inside the large bracket) in the dg derived category of abelian groups.

\section{Syntomic cohomology}

Let $\mathcal{O}_{K}$ be a complete discrete valuation ring with fraction field $K$ of characteristic 0 and with perfect residue field $k$ of characteristic $p$. Let $\varpi$ be a uniformizer of $\mathcal{O}_{K}$; we will keep it fixed throughout the paper. ${ }^{1}$ Let $W(k)$ be the ring of Witt vectors of $k$ with fraction field $F$ (that is, $\left.W(k)=\mathcal{O}_{F}\right)$; let $e$ be the ramification index of $K$ over $F$. Let $\sigma=\varphi$ be the absolute Frobenius morphism on $W(\bar{k})$. For an $\mathcal{O}_{K}$-scheme $X$, let $X_{0}$ denote the special fiber of $X$, and let $X_{n}$ denote the reduction modulo $p^{n}$ of $X$. We will denote by $\mathcal{O}_{K}, \mathcal{O}_{K}^{\times}$, and $\mathcal{O}_{K}^{0}$ the $\operatorname{scheme} \operatorname{Spec}\left(\mathcal{O}_{K}\right)$ with the trivial, canonical (that is, associated to the closed point), and ( $\mathbf{N} \rightarrow \mathcal{O}_{K}, 1 \mapsto 0$ ) log-structure, respectively.

In this section, we will briefly review the definitions of the syntomic and the syntomic-étale cohomology and their basic properties. For details, we refer the reader to [Tsu99, 2], [Tsu98].

\subsection{Syntomic cohomology}

For a $\log$-scheme $X$, we denote by $X_{\text {syn }}$ the small syntomic site of $X$. It is built from log-syntomic morphisms $f: Y \rightarrow Z$ in the sense of Kato [Kat94, 2.5] (see also [BM02, 6.1]); that is, the morphism $f$ is integral, the underlying morphism of schemes is flat and locally of finite presentation, and, étale locally on $Y$, there is a factorization $Y \stackrel{i}{\hookrightarrow} W \stackrel{h}{\rightarrow} Z$, where $h$ is log-smooth and $i$ is an exact closed immersion that is transversally regular over $Z$.

For a log-scheme $X$ log-syntomic over $\operatorname{Spec}(W(k))$, define

$$
\mathcal{O}_{n}^{\mathrm{cr}}(X)=H_{\mathrm{cr}}^{0}\left(X_{n}, \mathcal{O}_{X_{n}}\right), \quad \mathcal{J}_{n}^{[r]}(X)=H_{\mathrm{cr}}^{0}\left(X_{n}, \mathcal{J}_{X_{n}}^{[r]}\right)
$$

where $\mathcal{O}_{X_{n}}$ is the structure sheaf of the absolute crystalline site (that is, over $W_{n}(k)$ ) and $\mathcal{J}_{X_{n}}=$ $\operatorname{Ker}\left(\mathcal{O}_{X_{n} / W_{n}(k)} \rightarrow \mathcal{O}_{X_{n}}\right)$ and $\mathcal{J}_{X_{n}}^{[r]}$ is its $r$ th divided power. Set $\mathcal{J}_{X_{n}}^{[r]}=\mathcal{O}_{X_{n}}$ if $r \leqslant 0$. We know [FM87, II.1.3] that the presheaves $\mathcal{J}_{n}^{[r]}$ are sheaves on $X_{n, \text { syn }}$, flat over $\mathbf{Z} / p^{n}$, and that $\mathcal{J}_{n+1}^{[r]} \otimes \mathbf{Z} / p^{n} \simeq \mathcal{J}_{n}^{[r]}$. There is a natural functorial isomorphism

$$
H^{*}\left(X_{\mathrm{syn}}, \mathcal{J}_{n}^{[r]}\right) \simeq H_{\mathrm{cr}}^{*}\left(X_{n}, \mathcal{J}_{X_{n}}^{[r]}\right)
$$

that is compatible with the Frobenius morphism. It is easy to see that $\varphi\left(\mathcal{J}_{n}^{[r]}\right) \subset p^{r} \mathcal{O}_{n}^{\text {cr }}$ for $0 \leqslant r \leqslant p-1$. This fails in general, and we modify $\mathcal{J}_{n}^{[r]}$ :

$$
\mathcal{J}_{n}^{\langle r\rangle}:=\left\{x \in \mathcal{J}_{n+s}^{[r]} \mid \varphi(x) \in p^{r} \mathcal{O}_{n+s}^{\text {cr }}\right\} / p^{n} \quad \text { for some } s \geqslant r .
$$

This definition is independent of $s$. We check that $\mathcal{J}_{n}^{\langle r\rangle}$ is flat over $\mathbf{Z} / p^{n}$ and $\mathcal{J}_{n+1}^{\langle r\rangle} \otimes \mathbf{Z} / p^{n} \simeq \mathcal{J}_{n}^{\langle r\rangle}$. This allows us to define the divided Frobenius morphism $\varphi_{r}=" \varphi / p^{r "}: \mathcal{J}_{n}^{\langle r\rangle} \rightarrow \mathcal{O}_{n}^{\text {cr }}$.

\footnotetext{
${ }^{1}$ This is necessary to fix an embedding of $\operatorname{Spec}\left(\mathcal{O}_{K}\right)$ into a smooth scheme over $\mathbf{Z}_{p}$.
} 


\section{ERTL AND W. NiziOŁ}

Set

$$
\mathcal{S}_{n}(r):=\operatorname{Cone}\left(\mathcal{J}_{n}^{\langle r\rangle} \stackrel{1-\varphi_{r}}{\longrightarrow} \mathcal{O}_{n}^{\mathrm{cr}}\right)[-1]
$$

Since the sequence

$$
0 \longrightarrow \mathcal{S}_{n}(r) \longrightarrow \mathcal{J}_{n}^{\langle r\rangle} \stackrel{1-\varphi_{r}}{\longrightarrow} \mathcal{O}_{n}^{\text {cr }} \longrightarrow 0
$$

is exact, we actually have

$$
\mathcal{S}_{n}(r):=\operatorname{Ker}\left(\mathcal{J}_{n}^{\langle r\rangle} \stackrel{1-\varphi_{r}}{\longrightarrow} \mathcal{O}_{n}^{\text {cr }}\right) .
$$

In the same way, we can define syntomic sheaves $\mathcal{S}_{n}(r)$ on $X_{m \text {,syn }}$ for $m \geqslant n$. Abusing notation, we set $\mathcal{S}_{n}(r)=i_{*} \mathcal{S}_{n}(r)$ for the natural map $i: X_{m \text {,syn }} \rightarrow X_{\text {syn }}$. Since $i_{*}$ is exact, $H^{*}\left(X_{m, \text { syn }}, \mathcal{S}_{n}(r)\right)=$ $H^{*}\left(X_{\text {syn }}, \mathcal{S}_{n}(r)\right)$. Because of that, we will write $\mathcal{S}_{n}(r)$ for the syntomic sheaves on $X_{m, \text { syn }}$ as well as on $X_{\text {syn }}$. We will also need the "undivided" version of syntomic complexes of sheaves:

$$
\mathcal{S}_{n}^{\prime}(r):=\operatorname{Cone}\left(\mathcal{J}_{n}^{[r]} \stackrel{p^{r}-\varphi}{\longrightarrow} \mathcal{O}_{n}^{\text {cr }}\right)[-1] .
$$

For $r, i \geqslant 0$, we have the long exact sequences

$$
\begin{aligned}
& \rightarrow H^{i}\left(X_{\mathrm{syn}}, \mathcal{S}_{n}(r)\right) \rightarrow H_{\mathrm{cr}}^{i}\left(X_{n}, J_{X_{n}}^{\langle r\rangle}\right) \stackrel{1-\varphi_{r}}{\longrightarrow} H_{\mathrm{cr}}^{i}\left(X_{n}, \mathcal{O}_{X_{n}}\right) \rightarrow \\
& \rightarrow H^{i}\left(X_{\mathrm{syn}}, \mathcal{S}_{n}^{\prime}(r)\right) \rightarrow H_{\mathrm{cr}}^{i}\left(X_{n}, J_{X_{n}}^{[r]}\right) \stackrel{p^{r}-\varphi}{\longrightarrow} H_{\mathrm{cr}}^{i}\left(X_{n}, \mathcal{O}_{X_{n}}\right) \rightarrow .
\end{aligned}
$$

Proposition 2.1 ([CN17, Proposition 3.12]). For $X$ a fine and saturated log-smooth log-scheme over $\mathcal{O}_{K}^{\times}$and $0 \leqslant r \leqslant p-2$, the natural map of complexes of sheaves on the étale site of $X_{0}$

$$
\tau_{\leqslant r} \mathcal{S}_{n}(r) \rightarrow \mathcal{S}_{n}(r)
$$

is a quasi-isomorphism. For $X$ semistable over $\mathcal{O}_{K}$ and $r \geqslant 0$, the natural map of complexes of sheaves on the étale site of $X_{0}$

$$
\tau_{\leqslant r} \mathcal{S}_{n}^{\prime}(r) \rightarrow \mathcal{S}_{n}^{\prime}(r)
$$

is a $p^{N r}$-quasi-isomorphism for a universal constant $N$.

The natural map $\omega: \mathcal{S}_{n}^{\prime}(r) \rightarrow \mathcal{S}_{n}(r)$ induced by the maps $p^{r}: \mathcal{J}_{n}^{[r]} \rightarrow \mathcal{J}_{n}^{\langle r\rangle}$ and Id: $\mathcal{O}_{n}^{\text {cr }} \rightarrow \mathcal{O}_{n}^{\text {cr }}$ has kernel and cokernel killed by $p^{r}$. So does the map $\tau: \mathcal{S}_{n}(r) \rightarrow \mathcal{S}_{n}^{\prime}(r)$ induced by the maps Id $: \mathcal{J}_{n}^{\langle r\rangle} \rightarrow \mathcal{J}_{n}^{[r]}$ and $p^{r}: \mathcal{O}_{n}^{\text {cr }} \rightarrow \mathcal{O}_{n}^{\text {cr }}$. We have $\tau \omega=\omega \tau=p^{r}$.

If it does not cause confusion, we will write $\mathcal{S}_{n}(r)$ and $\mathcal{S}_{n}^{\prime}(r)$ also for $\mathrm{R} \varepsilon_{*} \mathcal{S}_{n}(r)$ and $\mathrm{R} \varepsilon_{*} \mathcal{S}_{n}^{\prime}(r)$,

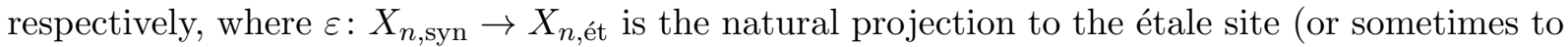
the Nisnevich site).

2.1.1 Syntomic cohomology and differential forms. Let $X$ be a syntomic scheme over $W(k)$. Recall the differential definition [Kat87] of syntomic cohomology. First, assume that we have an immersion $\iota: X \hookrightarrow Z$ over $W(k)$ such that $Z$ is a smooth $W(k)$-scheme endowed with a compatible system of liftings of the Frobenius morphism $\left\{F_{n}: Z_{n} \rightarrow Z_{n}\right\}$. Let $D_{n}=D_{X_{n}}\left(Z_{n}\right)$ be the PD-envelope of $X_{n}$ in $Z_{n}$ (compatible with the canonical PD-structure on $p W_{n}(k)$ ) and $J_{D_{n}}$ the ideal of $X_{n}$ in $D_{n}$. Set $J_{D_{n}}^{\langle r\rangle}:=\left\{a \in J_{D_{n+s}}^{[r]} \mid \varphi(a) \in p^{r} \mathcal{O}_{D_{n+s}}\right\} / p^{n}$ for some $s \geqslant r$. For $0 \leqslant r \leqslant p-1$, we have $J_{D_{n}}^{\langle r\rangle}=J_{D_{n}}^{[r]}$. This definition is independent of $s$. Consider the following complexes of sheaves on $X_{\text {ét }}$ :

$$
\begin{aligned}
& S_{n}(r)_{X, Z}:=\text { Cone }\left(J_{D_{n}}^{\langle r-\bullet} \otimes \Omega_{Z_{n}}^{\bullet} \stackrel{1-\varphi_{r}}{\longrightarrow} \mathcal{O}_{D_{n}} \otimes \Omega_{Z_{n}}^{\bullet}\right)[-1], \\
& S_{n}^{\prime}(r)_{X, Z}:=\text { Cone }\left(J_{D_{n}}^{[r-\bullet} \otimes \Omega_{Z_{n}}^{\bullet} \stackrel{p^{r}-\varphi}{\longrightarrow} \mathcal{O}_{D_{n}} \otimes \Omega_{Z_{n}}^{\bullet}[-1],\right.
\end{aligned}
$$




\section{Syntomic COHOMOLOGY AND p-ADIC MOTIVIC COHOMOLOGY}

where $\Omega_{Z_{n}}^{\bullet}:=\Omega_{Z_{n} / W_{n}(k)}^{\bullet}$ and $\varphi_{r}$ is " $\varphi / p^{r}$ " (see [Tsu99, 2.1] for details). The complexes $S_{n}(r)_{X, Z}$ and $S_{n}^{\prime}(r)_{X, Z}$ are, up to canonical quasi-isomorphisms, independent of the choice of $\iota$ and $\left\{F_{n}\right\}$ (and we will omit the subscript $Z$ from the notation). Again, the natural maps $\omega: S_{n}^{\prime}(r)_{X} \rightarrow$ $S_{n}(r)_{X}$ and $\tau: S_{n}(r)_{X} \rightarrow S_{n}^{\prime}(r)_{X}$ have kernels and cokernels annihilated by $p^{r}$.

In general, immersions as above exist étale locally, and we define $S_{n}(r)_{X} \in \mathbf{D}^{+}\left(X_{\text {ét }}, \mathbf{Z} / p^{n}\right)$ by gluing the local complexes. We define $S_{n}^{\prime}(r)_{X}$ in a similar way. There are natural quasiisomorphisms $S_{n}(r)_{X} \simeq \mathcal{S}_{n}(r)_{X}$ and $S_{n}^{\prime}(r)_{X} \simeq \mathcal{S}_{n}^{\prime}(r)_{X}$.

Now, let $X$ be a log-syntomic scheme over $W(k)$. Using log-crystalline cohomology, the above construction of syntomic complexes goes through almost verbatim (see [Tsu99, 2.1] for details) to yield the logarithmic analogs $S_{n}(r)$ and $S_{n}^{\prime}(r)$ on $X_{\text {ét }}$. In this paper, we are often interested in log-schemes coming from a regular syntomic scheme $X$ over $W(k)$ and a relative simple (that is, with no self-intersections) normal crossing divisor $D$ on $X$. In such cases, we will write $S_{n}(r)_{X}(D)$ and $S_{n}^{\prime}(r)_{X}(D)$ for the syntomic complexes and use the Zariski topology instead of the étale one.

2.1.2 Products. We need to discuss products. Assume that we are in the lifted situation (2.2). Then we have a product structure

$$
\cup: S_{n}^{\prime}(r)_{X, Z} \otimes S_{n}^{\prime}\left(r^{\prime}\right)_{X, Z} \rightarrow S_{n}^{\prime}\left(r+r^{\prime}\right)_{X, Z}, \quad r, r^{\prime} \geqslant 0,
$$

defined by the following formulas:

$$
\begin{gathered}
(x, y) \otimes\left(x^{\prime}, y^{\prime}\right) \mapsto\left(x x^{\prime},(-1)^{a} p^{r} x y^{\prime}+y \varphi\left(x^{\prime}\right)\right), \\
(x, y) \in S_{n}^{\prime}(r)_{X, Z}^{a}=\left(J_{D_{n}}^{[r-a]} \otimes \Omega_{Z_{n}}^{a}\right) \oplus\left(\mathcal{O}_{D_{n}} \otimes \Omega_{Z_{n}}^{a-1}\right), \\
\left(x^{\prime}, y^{\prime}\right) \in S_{n}^{\prime}\left(r^{\prime}\right)_{X, Z}^{b}=\left(J_{D_{n}}^{\left[r^{\prime}-b\right]} \otimes \Omega_{Z_{n}}^{b}\right) \oplus\left(\mathcal{O}_{D_{n}} \otimes \Omega_{Z_{n}}^{b-1}\right) .
\end{gathered}
$$

Globalizing, we obtain the product structure

$$
\cup: S_{n}^{\prime}(r)_{X} \otimes^{\mathbb{L}} S_{n}^{\prime}\left(r^{\prime}\right)_{X} \rightarrow S_{n}^{\prime}\left(r+r^{\prime}\right)_{X}, \quad r, r^{\prime} \geqslant 0 .
$$

This product is clearly compatible with the crystalline product (via the canonical map $S_{n}^{\prime}(r)_{X} \rightarrow$ $\left.J_{X_{n}}^{[r]}\right)$.

Similarly, we have the product structures

$$
\cup: S_{n}(r)_{X, Z} \otimes S_{n}\left(r^{\prime}\right)_{X, Z} \rightarrow S_{n}\left(r+r^{\prime}\right)_{X, Z}, \quad r, r^{\prime} \geqslant 0,
$$

defined by the formulas

$$
\begin{gathered}
(x, y) \otimes\left(x^{\prime}, y^{\prime}\right) \mapsto\left(x x^{\prime},(-1)^{a} x y^{\prime}+y \varphi_{r^{\prime}}\left(x^{\prime}\right)\right), \\
(x, y) \in S_{n}(r)_{X, Z}^{a}=\left(J_{D_{n}}^{\langle r-a\rangle} \otimes \Omega_{Z_{n}}^{a}\right) \oplus\left(\mathcal{O}_{D_{n}} \otimes \Omega_{Z_{n}}^{a-1}\right), \\
\left(x^{\prime}, y^{\prime}\right) \in S_{n}\left(r^{\prime}\right)_{X, Z}^{b}=\left(J_{D_{n}}^{\left\langle r^{\prime}-b\right\rangle} \otimes \Omega_{Z_{n}}^{b}\right) \oplus\left(\mathcal{O}_{D_{n}} \otimes \Omega_{Z_{n}}^{b-1}\right) .
\end{gathered}
$$

Globalizing, we obtain the product structure

$$
\cup: S_{n}(r)_{X} \otimes^{\mathbb{L}} S_{n}\left(r^{\prime}\right)_{X} \rightarrow S_{n}\left(r+r^{\prime}\right)_{X}, \quad r, r^{\prime} \geqslant 0 .
$$

This product is also clearly compatible with the crystalline product (via the canonical map $\left.S_{n}(r)_{X} \rightarrow J_{X_{n}}^{\langle r\rangle}\right)$.

The above product structures are compatible with the maps $\omega$. On the other hand, the maps $\tau$ are, in general, not compatible with products. 


\section{ERTL AND W. NizioŁ}

2.1.3 Symbol maps. Let $X$ be a regular syntomic scheme over $W(k)$ with a divisor $D$ with relative simple normal crossings. Recall that there are symbol maps defined by Kato and Tsuji [Tsu99, 2.2]

$$
\left(M_{X, n}^{\mathrm{gp}}\right)^{\otimes r} \rightarrow H^{r}\left(S_{n}^{\prime}(r)_{X}(D)\right), \quad\left(M_{X, n+1}^{\mathrm{gp}}\right)^{\otimes r} \rightarrow H^{r}\left(S_{n}(r)_{X}(D)\right), \quad r \geqslant 0,
$$

where, for a log-scheme $X$, its log-structure is denoted by $M_{X}$. For $r=1$, we get the first Chern class maps (recall that $M_{X}^{\mathrm{gp}}=j_{*} \mathcal{O}_{X \backslash D}^{*}$, where $j: X \backslash D \hookrightarrow X$ is the natural immersion)

$$
\begin{gathered}
c_{1}^{\mathrm{syn}}: j_{*} \mathcal{O}_{X \backslash D}^{*}[-1] \rightarrow i_{*} j_{*} \mathcal{O}_{(X \backslash D)_{n+1}}^{*}[-1] \rightarrow S_{n}(1)_{X}(D), \\
c_{1}^{\mathrm{syn}}: j_{*} \mathcal{O}_{X \backslash D}^{*}[-1] \rightarrow i_{*} j_{*} \mathcal{O}_{(X \backslash D)_{n}}^{*}[-1] \rightarrow S_{n}^{\prime}(1)_{X}(D),
\end{gathered}
$$

that are compatible; that is, the following diagram commutes:

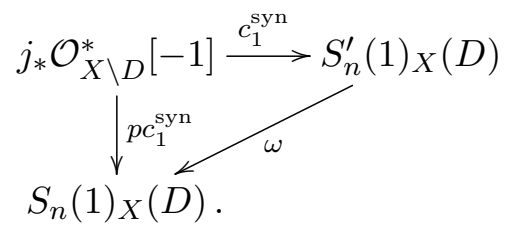

In the embedded situation, these classes are defined in the following way. Let $C_{n}$ be the complex

$$
\left(1+J_{D_{n}} \rightarrow M_{D_{n}}^{\mathrm{gp}}\right) \simeq j_{*} \mathcal{O}_{(X \backslash D)_{n}}^{*}[-1] \simeq M_{X_{n}}^{\mathrm{gp}}[-1] .
$$

The Chern class maps

$$
c_{1}^{\mathrm{syn}}: j_{*} \mathcal{O}_{(X \backslash D)_{n}}^{*}[-1] \rightarrow S_{n}^{\prime}(1)_{X}(D), \quad c_{1}^{\mathrm{syn}}: j_{*} \mathcal{O}_{(X \backslash D)_{n+1}}^{*}[-1] \rightarrow S_{n}(1)_{X}(D)
$$

are defined by the morphisms of complexes

$$
C_{n} \rightarrow S_{n}^{\prime}(1)_{X, Z}, \quad C_{n+1} \rightarrow S_{n}(1)_{X, Z}
$$

given by the formulas

$$
\begin{aligned}
& 1+J_{D_{n}} \rightarrow\left(S_{n}^{\prime}(1)_{X, Z}\right)^{0}=J_{D_{n}}, \quad a \mapsto \log a, \\
& 1+J_{D_{n+1}} \rightarrow\left(S_{n}(1)_{X, Z}\right)^{0}=J_{D_{n}}, \quad a \mapsto \log a \bmod p^{n}
\end{aligned}
$$

and

$$
\begin{aligned}
M_{D_{n}}^{\mathrm{gp}} \rightarrow\left(S_{n}^{\prime}(1)_{X, Z}\right)^{1}=\left(\mathcal{O}_{D_{n}} \otimes \Omega_{Z_{n}}^{1}\right) \oplus \mathcal{O}_{D_{n}}, \quad b \mapsto\left(d \log b, \log \left(b^{p} \varphi_{D_{n}}(b)^{-1}\right)\right), \\
M_{D_{n+1}}^{\mathrm{gp}} \rightarrow\left(S_{n}(1)_{X, Z}\right)^{1}=\left(\mathcal{O}_{D_{n}} \otimes \Omega_{Z_{n}}^{1}\right) \oplus \mathcal{O}_{D_{n}}, \quad b \mapsto\left(d \log b \bmod p^{n}, p^{-1} \log \left(b^{p} \varphi_{D_{n+1}}(b)^{-1}\right)\right) .
\end{aligned}
$$

The symbol maps (2.3) for general $r$ are obtained from the case $r=1$ using the product structure on syntomic cohomology.

\subsection{Syntomic-étale cohomology}

We will now recall the definition and basic properties of syntomic-étale cohomology. The relationship between syntomic cohomology and syntomic-étale cohomology mirrors the one between étale nearby cycles and étale cohomology. Let $X$ be a log-scheme, log-syntomic over $\operatorname{Spec}(W(k))$. We will need the logarithmic version of the syntomic-étale site of Fontaine-Messing [FM87]. We say that a morphism $\mathcal{Z} \rightarrow \mathcal{Y}$ of $p$-adic formal $\log$-schemes over $\operatorname{Spf}(W(k))$ is (small) log-syntomic (see [Tsu98] for a precise definition) if every $Z_{n} \rightarrow Y_{n}$ is (small) $\log$-syntomic. For a formal $\log$ scheme $\mathcal{Z}$, the syntomic-étale site $\mathcal{Z}_{\text {sé }}$ is defined by taking as objects morphisms $f: \mathcal{Y} \rightarrow \mathcal{Z}$ that are small log-syntomic and have log-étale generic fiber. This last condition means that, étale 


\section{Syntomic COHOMOLOGY AND p-ADIC MOTIVIC COHOMOLOGY}

locally on $\mathcal{Y}$, the morphism $f$ has a factorization $\mathcal{Y} \stackrel{i}{\rightarrow} \mathcal{X} \stackrel{g}{\rightarrow} \mathcal{Z}$ with $\mathcal{X}$ affine, $i$ an exact closed immersion, and $g$ log-smooth such that the map $F \otimes_{W(k)} \Gamma\left(\mathcal{Y}, I / I^{2}\right) \rightarrow F \otimes_{W(k)} \Gamma\left(\mathcal{Y}, i^{*} \Omega_{\mathcal{X} / \mathcal{Z}}^{1}\right)$ is an isomorphism, where $I$ is the ideal of $\mathcal{O}_{\mathcal{X}}$ defining $\mathcal{Y}$. For a $\log$-scheme $Z$, we also have the syntomic-étale site $Z_{\text {sé }}$. Here the objects are morphisms $U \rightarrow Z$ that are small log-syntomic with the generic fiber $U_{K}$ log-étale over $Z_{K}$.

Let $\widehat{X}$ be the $p$-adic completion of $X$. Let $i: X_{n \text {,ét }} \rightarrow X_{\text {ét }}$ and $j: X_{\mathrm{tr}, K \text {,ét }} \rightarrow X_{\text {ét }}$ be the natural maps. Here $X_{\operatorname{tr}}$ is the open set of $X$ where the log-structure is trivial. We have the following commutative diagram of maps of topoi:

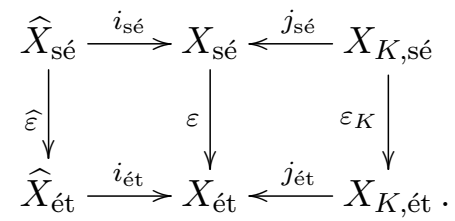

First, assume $0 \leqslant r \leqslant p-2$. Abusively, let $\mathcal{S}_{n}(r)$ denote also the direct image of $\mathcal{S}_{n}(r)$ under the canonical morphism $X_{n, \text { syn }} \rightarrow \widehat{X}_{\text {sé. }}$ By [FM87, III.5], for $j^{\prime}: X_{\mathrm{tr}, K \text {,ét }} \rightarrow X_{K \text {,sé, there is a canonical }}$ homomorphism

$$
\alpha_{r}: \mathcal{S}_{n}(r) \rightarrow i_{\text {sé }}^{*} j_{\text {sé } *} j_{*}^{\prime} G \mathbf{Z} / p^{n}(r),
$$

where $G$ denotes the Godement resolution of a sheaf (or a complex of sheaves). Similarly, for any $r \geqslant 0$, we get a natural map

$$
\tilde{\alpha}_{r}: \mathcal{S}_{n}(r) \rightarrow i_{\text {sé }}^{*} j_{\text {sé } *} j_{*}^{\prime} G \mathbf{Z} / p^{n}(r)^{\prime},
$$

where $\mathbf{Z} / p^{n}(r)^{\prime}=\left(p^{a} a !\right)^{-1} \mathbf{Z} / p^{n}(r)$ if $r=(p-1) a+b$ with $a, b \in \mathbf{Z}$ and $0 \leqslant b<p-1$ [FM87, III.5]. Composing with the map $\mathcal{S}_{n}^{\prime}(r) \rightarrow \mathcal{S}_{n}(r)$, we get a natural morphism

$$
\alpha_{r}: \mathcal{S}_{n}^{\prime}(r) \rightarrow i_{\text {sé }}^{*} j_{\text {sé } *} j_{*}^{\prime} G \mathbf{Z} / p^{n}(r)^{\prime} .
$$

2.2.1 Syntomic complexes and $p$-adic nearby cycles. For log-schemes over $\mathcal{O}_{K}^{\times}$, in a stable range, syntomic cohomology tends to compute (via the period morphism) $p$-adic nearby cycles. We will briefly recall the relevant theorems. For $0 \leqslant r \leqslant p-2$, there is a natural homomorphism on the étale site of $X_{n}$,

$$
\alpha_{r}: \mathcal{S}_{n}(r) \rightarrow i^{*} \mathrm{R} j_{*} \mathbf{Z} / p^{n}(r) .
$$

To define it, we apply $\mathrm{R} \widehat{\varepsilon}_{*}$ to the map $\mathcal{S}_{n}(r) \rightarrow i_{\text {sé }}^{*} \mathrm{R} j_{\text {sé } *} \mathrm{R} j_{*}^{\prime} \mathbf{Z} / p^{n}(r)$ induced from the map $\alpha_{r}$ described above and get

$$
\mathrm{R} \varepsilon_{*} \mathcal{S}_{n}(r)=\mathrm{R} \widehat{\varepsilon}_{*} \mathcal{S}_{n}(r) \rightarrow \mathrm{R} \widehat{\varepsilon}_{*} i_{\text {sé }}^{*} \mathrm{R} j_{\text {sé } *} \mathrm{R} j_{*}^{\prime} \mathbf{Z} / p^{n}(r)=i_{\text {ét }}^{*} \mathrm{R} \varepsilon_{*} \mathrm{R} j_{\text {sé } *} \mathrm{R} j_{*}^{\prime} \mathbf{Z} / p^{n}(r)=i^{*} \mathrm{R} j_{*} \mathbf{Z} / p^{n}(r) .
$$

The first equality follows from the fact that the morphism $X_{n, \text { syn }} \rightarrow \widehat{X}_{\text {sé }}$ is exact [FM87, III.4.1]. The second equality was proved in [KM92, 2.5], [Tsu98, 5.2.3]. One checks that $\alpha_{r}$ is compatible with products.

Theorem 2.2 ([Tsu00, Theorem 5.1]). For $i \leqslant r \leqslant p-2$ and for a fine and saturated logscheme $X$ log-smooth over $\mathcal{O}_{K}^{\times}$, the period map

$$
\alpha_{r}: \mathcal{S}_{n}(r)_{X} \stackrel{\sim}{\rightarrow} \tau_{\leqslant r} i^{*} \mathrm{R} j_{*} \mathbf{Z} / p^{n}(r)_{X_{\mathrm{tr}}} .
$$

is an isomorphism.

Note that the definition of the period map in [Tsu00] is different from the one in [Tsu98] that we use here. However, they clearly agree in the derived category. 


\section{ERTL AND W. NiziOŁ}

Similarly, for any $r \geqslant 0$, we get a natural map

$$
\tilde{\alpha}_{r}: \mathcal{S}_{n}(r) \rightarrow i^{*} \mathrm{R} j_{*} \mathbf{Z} / p^{n}(r)^{\prime} .
$$

Composing with the map $\omega: \mathcal{S}_{n}^{\prime}(r) \rightarrow \mathcal{S}_{n}(r)$, we get a natural morphism, compatible with products,

$$
\alpha_{r}: \mathcal{S}_{n}^{\prime}(r) \rightarrow i^{*} \mathrm{R} j_{*} \mathbf{Z} / p^{n}(r)^{\prime} .
$$

Theorem 2.3 ([CN17, Theorem 1.1]). For $0 \leqslant i \leqslant r$ and for a semistable scheme $X$ over $\mathcal{O}_{K}$, consider the period map

$$
\alpha_{r}: \mathcal{H}^{i}\left(\mathcal{S}_{n}^{\prime}(r)_{X}\right) \rightarrow i^{*} \mathrm{R}^{i} j_{*} \mathbf{Z} / p^{n}(r)_{X_{\mathrm{tr}}}^{\prime} .
$$

If $K$ has enough roots of unity, then the kernel and cokernel of this map are annihilated by $p^{N r}$ for a universal constant $N$ depending only on $p$ (and $\operatorname{dim} X$ if $p=2$ ). In general, the kernel and cokernel of this map are annihilated by $p^{N r}$ for an integer $N=N(e, p)$ which depends only on $e$ and $p$.

2.2.2 Syntomic-étale cohomology. Recall [FM87, III.4.4], [Tsu98, 5.2.2] that the functor $\mathcal{F} \mapsto$ $\left(i_{\text {sé }}^{*} \mathcal{F}, j_{\text {sé }}^{*} \mathcal{F}, i_{\text {sé }}^{*} \mathcal{F} \rightarrow i_{\text {sé }}^{*} j_{\text {sé } *} j_{\text {sé }}^{*} \mathcal{F}\right)$ from the category of sheaves on $X_{\text {sé }}$ to the category of triples $\left(\mathcal{G}, \mathcal{H}, \mathcal{G} \rightarrow i_{\text {sé }}^{*} j_{\text {sé } *} \mathcal{H}\right)$, where $\mathcal{G}$ and $\mathcal{H}$ are sheaves on $\widehat{X}_{\text {sé }}$ and $X_{K \text {,sé }}$, respectively, is an equivalence of categories. It follows that we can glue the complexes of sheaves $\mathcal{S}_{n}(r)$ and $\mathcal{S}_{n}^{\prime}(r)$ and the complexes of sheaves $j_{*}^{\prime} G \mathbf{Z} / p^{n}(r)$ and $j_{*}^{\prime} G \mathbf{Z} / p^{n}(r)^{\prime}$ by the maps $\alpha_{r}$ and obtain complexes of sheaves $\mathcal{E}_{n}(r)$ and $\mathcal{E}_{n}^{\prime}(r)$ on $X_{\text {sé }}$. We have the exact sequences

$$
\begin{aligned}
& 0 \rightarrow j_{\text {sé } !} j_{*}^{\prime} G \mathbf{Z} / p^{n}(r) \rightarrow \mathcal{E}_{n}(r) \rightarrow i_{*} \mathcal{S}_{n}(r) \rightarrow 0, \quad 0 \leqslant r \leqslant p-2, \\
& 0 \rightarrow j_{\text {sé } ! j_{*}^{\prime}} G \mathbf{Z} / p^{n}(r)^{\prime} \rightarrow \mathcal{E}_{n}^{\prime}(r) \rightarrow i_{*} \mathcal{S}_{n}^{\prime}(r) \rightarrow 0, \quad r \geqslant 0 .
\end{aligned}
$$

Remark 2.4. The syntomic-étale complexes $\mathcal{E}_{n}(r)$ that we describe here are the same (in the derived category) as those defined by Fontaine-Messing in [FM87, 5] in the case when $X_{\mathrm{tr}}=X$ but differ from those defined by Tsuji in [Tsu98, 5.2] in the general situation. More specifically, we have

$$
\mathcal{E}_{n}^{T}(r)=\mathcal{H}^{0}\left(\mathcal{E}_{n}(r)\right)
$$

where we write $\mathcal{E}_{n}^{T}(r)$ for the syntomic-étale sheaves of Tsuji.

If it does not cause confusion, we will denote by $\mathcal{E}_{n}(r)$ and $\mathcal{E}_{n}^{\prime}(r)$ also the derived pushforwards of $\mathcal{E}_{n}(r)$ and $\mathcal{E}_{n}^{\prime}(r)$ to $X_{\text {ét }}$. Note that they are quasi-isomorphic to the complexes obtained by gluing the complexes of sheaves $\mathcal{S}_{n}(r)$ and $\mathcal{S}_{n}^{\prime}(r)$ and the complexes of sheaves $j_{*}^{\prime} G \mathbf{Z} / p^{n}(r)^{\prime}$ by the maps $\tilde{\alpha}_{r}$ and $\alpha_{r}$. Hence, we have the distinguished triangles

$$
j_{\text {ét! }} \mathrm{R} j_{*}^{\prime} \mathbf{Z} / p^{n}(r)^{\prime} \rightarrow \mathcal{E}_{n}(r) \rightarrow i_{*} \mathcal{S}_{n}(r), \quad j_{\text {ét! }} \mathrm{R} j_{*}^{\prime} \mathbf{Z} / p^{n}(r)^{\prime} \rightarrow \mathcal{E}_{n}^{\prime}(r) \rightarrow i_{*} \mathcal{S}_{n}^{\prime}(r),
$$

where $j^{\prime}: X_{\mathrm{tr}, K} \rightarrow X_{K}$, as well as the natural maps

$$
\tilde{\alpha}_{r}: \mathcal{E}_{n}(r) \rightarrow \mathrm{R} j_{*} \mathbf{Z} / p^{n}(r)^{\prime}, \quad \alpha_{r}: \mathcal{E}_{n}(r)^{\prime} \rightarrow \mathrm{R} j_{*} \mathbf{Z} / p^{n}(r)^{\prime}
$$

compatible with the maps $\tilde{\alpha}_{r}$ and $\alpha_{r}$. For $a \geqslant 0$, we have the truncated version of the above: the distinguished triangles

$$
\begin{aligned}
& j_{\text {ét! }} \tau_{\leqslant a} \mathrm{R} j_{*}^{\prime} \mathbf{Z} / p^{n}(r)^{\prime} \rightarrow \tau_{\leqslant a} \mathcal{E}_{n}(r) \rightarrow i_{*} \tau_{\leqslant a} \mathcal{S}_{n}(r) \text {, } \\
& j_{\text {ét! }} \tau_{\leqslant a} \mathrm{R} j_{*}^{\prime} \mathbf{Z} / p^{n}(r)^{\prime} \rightarrow \tau_{\leqslant a} \mathcal{E}_{n}^{\prime}(r) \rightarrow i_{*} \tau_{\leqslant a} \mathcal{S}_{n}^{\prime}(r) \text {. }
\end{aligned}
$$




\section{SyNTOMIC COHOMOLOGY AND $p$-ADIC MOTIVIC COHOMOLOGY}

2.2.3 Syntomic-étale cohomology and étale cohomology of the generic fiber. For a log-scheme over $\mathcal{O}_{K}^{\times}$, in a stable range, syntomic-étale cohomology tends to compute étale cohomology of the generic fiber.

Theorem 2.5. Let $X$ be a log-scheme $\log$-smooth over $\mathcal{O}_{K}^{\times}$. Let $j: X_{\operatorname{tr}} \hookrightarrow X$ be the natural open immersion. Then

(i) we have a natural quasi-isomorphism

$$
\tilde{\alpha}_{r}: \tau_{\leqslant r} \mathcal{E}_{n}(r) \simeq \tau_{\leqslant r} \mathrm{R} j_{*} \mathbf{Z} / p^{n}(r), \quad 0 \leqslant r \leqslant p-2 ;
$$

(ii) if $X$ is semistable, there exist a constant $N$ as in Theorem 2.3 and a natural morphism

$$
\alpha_{r}: \mathcal{E}_{n}^{\prime}(r) \rightarrow \mathrm{R} j_{*} \mathbf{Z} / p^{n}(r)^{\prime}, \quad r \geqslant 0,
$$

such that the induced map on cohomology sheaves in degree $q \leqslant r$ has kernel and cokernel annihilated by $p^{N r}$.

Proof. Assume $0 \leqslant r \leqslant p-2$. Consider the following commutative diagram of distinguished triangles:

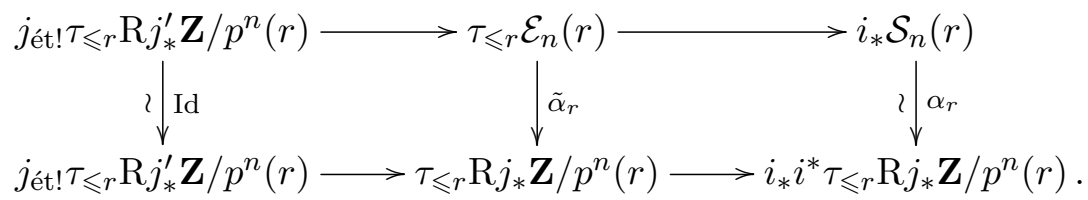

The top triangle is distinguished because we have the distinguished triangle from (2.7) and the natural map $\tau_{\leqslant r} \mathcal{S}_{n}(r) \stackrel{\sim}{\rightarrow} \mathcal{S}_{n}(r)$ is a quasi-isomorphism. The map $\alpha_{r}$ is a quasi-isomorphism by Theorem 2.2. The first part of the theorem follows.

For the second part, consider the following commutative diagram of distinguished triangles:

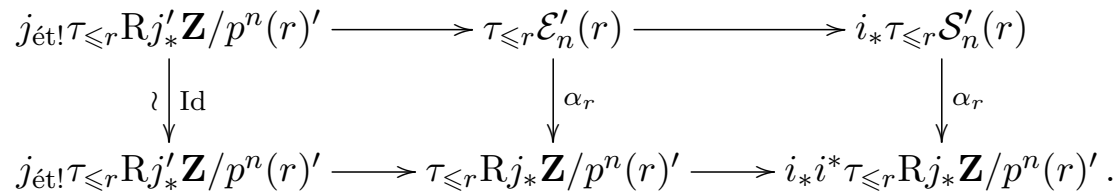

By Theorem 2.3, on the level of cohomology, the right period map $\alpha_{r}$ has kernels and cokernels killed by $p^{N r}$ for a constant $N$ as in the theorem. Hence, the same is true of the left map $\alpha_{r}$, as wanted.

Theorem 2.5 implies that the logarithmic syntomic-étale cohomology is close to the logarithmic syntomic-étale cohomology of the complement of the divisor at infinity.

Corollary 2.6. Let $X$ be a semistable scheme over $\mathcal{O}_{K}$ with a divisor at infinity $D_{\infty}$. We treat it is as a $\log$-scheme over $\mathcal{O}_{K}^{\times}$. Let $Y:=X \backslash D_{\infty}$, and let $j_{1}: Y \hookrightarrow X$.

(i) We have a natural quasi-isomorphism

$$
\tilde{\alpha}_{r}: \tau_{\leqslant r} \mathcal{E}_{n}(r)_{X} \stackrel{\sim}{\rightarrow} \tau_{\leqslant r} \mathrm{R} j_{1 *} \mathcal{E}_{n}(r)_{Y}, \quad 0 \leqslant r \leqslant p-2 .
$$

(ii) There exist a constant $N$ as in Theorem 2.3 and a natural morphism

$$
\alpha_{r}: \mathcal{E}_{n}^{\prime}(r)_{X} \rightarrow \mathrm{R} j_{1 *} \mathcal{E}_{n}^{\prime}(r)_{Y}, \quad r \geqslant 0,
$$

such that the induced map on cohomology sheaves in degree $q \leqslant r$ has kernel and cokernel annihilated by $p^{N r}$. 


\section{ERTL AND W. NiZIOE}

Proof. Note that $X_{\mathrm{tr}}=Y_{K}$, and set $j_{2}: Y_{K} \hookrightarrow Y$. We have $j=j_{1} j_{2}$. By Theorem 2.5, both terms in the first claim are quasi-isomorphic to

$$
\tau_{\leqslant r} \mathrm{R} j_{*} \mathbf{Z} / p^{n}(r)_{X_{\text {tr }}}=\tau_{\leqslant r} \mathrm{R} j_{1 *} \tau_{\leqslant r} \mathrm{R} j_{2 *} \mathbf{Z} / p^{n}(r)_{Y_{K}} .
$$

Hence, they are quasi-isomorphic. The second claim of the corollary is proved in the same way.

2.2.4 Nisnevich syntomic-étale cohomology. We will now pass to the Nisnevich topos of $X$. Denote the natural projection by $\varepsilon: X_{\text {ét }} \rightarrow X_{\mathrm{Nis}}$. For $r \geqslant 0$, by applying R $\varepsilon_{*}$ to the étale period map above and using that ${ }^{2} \mathrm{R} \varepsilon_{*} i^{*}=i^{*} \mathrm{R} \varepsilon_{*}$ (cf. [Gei04, 2.2.b]), we obtain a natural map

$$
\tilde{\alpha}_{r}: \mathrm{R} \varepsilon_{*} \mathcal{S}_{n}(r) \rightarrow i^{*} \mathrm{R} j_{*} \mathrm{R} \varepsilon_{*} \mathbf{Z} / p^{n}(r)^{\prime} .
$$

Composing with the map $\omega: \mathrm{R} \varepsilon_{*} \mathcal{S}_{n}^{\prime}(r) \rightarrow \mathrm{R} \varepsilon_{*} \mathcal{S}_{n}(r)$, we get a natural morphism, compatible with products,

$$
\alpha_{r}: \mathrm{R} \varepsilon_{*} \mathcal{S}_{n}^{\prime}(r) \rightarrow i^{*} \mathrm{R} j_{*} \mathrm{R} \varepsilon_{*} \mathbf{Z} / p^{n}(r)^{\prime} .
$$

Write, for simplicity, $\mathcal{S}_{n}(r)$ and $\mathcal{S}_{n}^{\prime}(r)$ for the derived pushforwards of $\mathcal{S}_{n}(r)$ and $\mathcal{S}_{n}^{\prime}(r)$ from $X_{\text {ét }}$ to $X_{\mathrm{Nis}}$, and likewise for $\mathcal{E}_{n}(r)$ and $\mathcal{E}_{n}^{\prime}(r)$. Notice that they are quasi-isomorphic to the complexes obtained by gluing the complexes of sheaves $\mathcal{S}_{n}(r)$ and $\mathcal{S}_{n}^{\prime}(r)$ on $X_{0, \mathrm{Nis}}$ and the complexes of sheaves $\varepsilon_{*} j_{*}^{\prime} G \mathbf{Z} / p^{n}(r)^{\prime}$ on $X_{K, \text { Nis }}$ by the maps $\tilde{\alpha}_{r}$ and $\alpha_{r}$. Hence, we have the distinguished triangles

$$
j_{\mathrm{Nis} !} \mathrm{R} j_{*}^{\prime} \mathrm{R} \varepsilon_{*} \mathbf{Z} / p^{n}(r)^{\prime} \rightarrow \mathcal{E}_{n}(r) \rightarrow i_{*} \mathcal{S}_{n}(r), \quad j_{\mathrm{Nis} !} \mathrm{R} j_{*}^{\prime} \mathrm{R} \varepsilon_{*} \mathbf{Z} / p^{n}(r)^{\prime} \rightarrow \mathcal{E}_{n}^{\prime}(r) \rightarrow i_{*} \mathcal{S}_{n}^{\prime}(r),
$$

as well as the natural maps

$$
\tilde{\alpha}_{r}: \mathcal{E}_{n}(r) \rightarrow \mathrm{R} j_{*} \mathrm{R} \varepsilon_{*} \mathbf{Z} / p^{n}(r)^{\prime}, \quad \alpha_{r}: \mathcal{E}_{n}(r)^{\prime} \rightarrow \mathrm{R} j_{*} \mathrm{R} \varepsilon_{*} \mathbf{Z} / p^{n}(r)^{\prime},
$$

compatible with the maps $\tilde{\alpha}_{r}$ and $\alpha_{r}$. For $a \geqslant 0$, we have the truncated version of the above: the distinguished triangles

$$
\begin{aligned}
& j_{\mathrm{Nis} !} \tau_{\leqslant a} \mathrm{R} j_{*}^{\prime} \mathrm{R} \varepsilon_{*} \mathbf{Z} / p^{n}(r)^{\prime} \rightarrow \tau_{\leqslant a} \mathcal{E}_{n}(r) \rightarrow i_{*} \tau_{\leqslant a} \mathcal{S}_{n}(r), \\
& j_{\mathrm{Nis} !} \tau_{\leqslant a} \mathrm{R} j_{*}^{\prime} \mathrm{R} \varepsilon_{*} \mathbf{Z} / p^{n}(r)^{\prime} \rightarrow \tau_{\leqslant a} \mathcal{E}_{n}^{\prime}(r) \rightarrow i_{*} \tau_{\leqslant a} \mathcal{S}_{n}^{\prime}(r) .
\end{aligned}
$$

Define the following complexes of sheaves on $X_{\mathrm{Nis}}$ :

$$
\begin{array}{ll}
\mathcal{S}_{n}(r)_{\mathrm{Nis}}:=\tau_{\leqslant r} \mathcal{S}_{n}(r), & \mathcal{S}_{n}^{\prime}(r)_{\mathrm{Nis}}:=\tau_{\leqslant r} \mathcal{S}_{n}^{\prime}(r), \\
\mathcal{E}_{n}(r)_{\mathrm{Nis}}:=\tau_{\leqslant r} \mathcal{E}_{n}(r), \quad & \mathcal{E}_{n}^{\prime}(r)_{\mathrm{Nis}}:=\tau_{\leqslant r} \mathcal{E}_{n}^{\prime}(r) .
\end{array}
$$

Example 2.7. For $X=\operatorname{Spec}(W(k))$, we have

$$
H^{i}\left(W(k), \mathcal{S}_{n}(r)_{\mathrm{Nis}}\right)= \begin{cases}\mathbf{Z} / p^{n}, & i=r=0, \\ W_{n}(k), & i=1, r \geqslant 1, \\ 0 & \text { otherwise } .\end{cases}
$$

Moreover, the morphism $H^{i}\left(W(k), \mathcal{E}_{n}(r)_{\mathrm{Nis}}\right) \rightarrow H^{i}\left(W(k), \mathcal{S}_{n}(r)_{\mathrm{Nis}}\right)$ is an isomorphism.

To see the first claim, note that we have

$$
\mathcal{S}_{n}(0)_{\text {ét }}: W_{n}(k) \stackrel{1-\varphi}{\longrightarrow} W_{n}(k), \quad \mathcal{S}_{n}(r)_{\text {ét }}: 0 \rightarrow W_{n}(k), \quad r \geqslant 1 .
$$

It follows that

$$
\mathcal{S}_{n}(0)_{\mathrm{Nis}}=\mathbf{Z} / p^{n}, \quad \mathcal{S}_{n}(r)_{\text {ét }}:=W_{n}(k)[-1], \quad r \geqslant 1 .
$$

\footnotetext{
${ }^{2}$ This equality fails for the projection to Zariski topology and is the reason we use Nisnevich topology instead of Zariski topology.
} 


\section{SyNTOMIC COHOMOLOGY AND $p$-ADIC MOTIVIC COHOMOLOGY}

For the second claim, use the distinguished triangle (2.9) and the fact that

$$
H^{i}\left(W(k), j_{\mathrm{Nis} !} \tau_{\leqslant a} \mathrm{R} j_{*}^{\prime} \mathrm{R} \varepsilon_{*} \mathbf{Z} / p^{n}(r)\right)=0, \quad i \geqslant 0,
$$

because $W(k)$ is Henselian.

\section{Syntomic cohomology and motivic cohomology}

\subsection{Syntomic cohomology and motivic cohomology}

This is the main section of this paper. We will prove Theorem 1.2 from the introduction.

3.1.1 Definition of motivic cohomology. Let $X$ be a smooth scheme over $\mathcal{O}_{K}$. Let $\mathbf{Z}(r)_{\mathrm{M}}$ denote the complex of motivic sheaves $\mathbf{Z}(r)_{\mathrm{M}}:=X \mapsto z^{r}(X, 2 r-*)$ in the étale topology of $X$. Let $\mathbf{Z} / p^{n}(r)_{\mathrm{M}}:=\mathbf{Z}(r)_{\mathrm{M}} \otimes \mathbf{Z} / p^{n}$. Recall how the complex $z^{r}(X, *)$ is defined [Blo86]. Denote by $\triangle^{n}$ the algebraic n-simplex Spec $\mathbf{Z}\left[t_{0}, \ldots, t_{n}\right] /\left(\sum t_{i}-1\right)$. Let $z^{r}(X, i)$ be the free abelian group generated by closed integral subschemes of codimension $r$ of $X \times \triangle^{i}$ meeting all faces properly. Then $z^{r}(X, *)$ is the chain complex thus defined, with boundaries given by pullbacks of cycles along face maps. This complex is covariant for proper morphisms (with a shift in weight and degree) and contravariant for flat morphisms.

We know that in the Zariski topology, $H^{j}\left(X_{\mathrm{Zar}}, \mathbf{Z} / p^{n}(i)_{\mathrm{M}}\right)=H^{j} \Gamma\left(X_{\mathrm{Zar}}, \mathbf{Z} / p^{n}(r)_{\mathrm{M}}\right)$ is the Bloch higher Chow group [Gei04, Theorem 3.2] and that this is also the case for the Nisnevich topology [Gei04, Proposition 3.6]. Locally, in the étale topology, when $p$ is invertible, the étale cycle class map defines a quasi-isomorphism $\mathbf{Z} / p^{n}(r)_{\mathrm{M}} \simeq \mathbf{Z} / p^{n}(r)$; when $X$ is of characteristic $p$, the logarithmic de Rham-Witt cycle class map defines a quasi-isomorphism $\mathbf{Z} / p^{n}(r)_{\mathrm{M}} \simeq W_{n} \Omega_{X, \log }^{r}[-r]$ (see [GL00]), where, for a log-scheme $Y$, the right-hand side $W_{n} \Omega_{Y, \log }^{*}$ denotes the sheaf of logarithmic de Rham-Witt differential forms [Lor02]. Moreover, if $i: Z \hookrightarrow X$ is a closed subscheme of codimension $c$ with open complement $j: U \hookrightarrow X$, then the exact sequence

$$
0 \rightarrow i_{*} \mathbf{Z}(r-c)_{M, Z}[-2 c] \rightarrow \mathbf{Z}(r)_{M, X} \rightarrow j_{*} \mathbf{Z}(r)_{M, U}
$$

forms a distinguished triangle in the derived category of sheaves on $X_{*}$, where $*$ denotes the Zariski or Nisnevich topology. We define the motivic cohomology as

$$
\begin{aligned}
H_{\mathrm{M}}^{*}\left(X, \mathbf{Z} / p^{n}(r)\right) & :=H^{*}\left(X_{\mathrm{Zar}}, \mathbf{Z} / p^{n}(r)_{M}\right)=H^{*}\left(X_{\mathrm{Nis}}, \mathbf{Z} / p^{n}(r)_{M}\right), \\
H_{\mathrm{M}, e ́ t}^{*}\left(X, \mathbf{Z} / p^{n}(r)\right) & :=H^{*}\left(X_{\text {ét }}, \mathbf{Z} / p^{n}(r)_{M}\right) .
\end{aligned}
$$

For a smooth scheme $Y$ over $\mathcal{O}_{K}$, we define its $p$-adic motivic cohomology as

$$
H_{\mathrm{M}}^{*}\left(Y, \mathbf{Q}_{p}(r)\right):=H^{*}\left(\operatorname{holim}_{n} \mathrm{R} \Gamma\left(Y_{\mathrm{Zar}}, \mathbf{Z} / p^{n}(r)_{\mathrm{M}}\right) \otimes \mathbf{Q}\right)=H^{*}\left(\operatorname{holim}_{n} \Gamma\left(Y_{\mathrm{Zar}}, \mathbf{Z} / p^{n}(r)_{\mathrm{M}}\right) \otimes \mathbf{Q}\right) .
$$

We define its étale version $H_{\mathrm{M} \text {,ét }}^{*}\left(Y, \mathbf{Q}_{p}(r)\right)$ in an analogous way.

3.1.2 $p^{N}$-homological algebra. We will need to control denominators. For that purpose, we introduce a few, very ad hoc, definitions and list a few of properties that we will use.

Definition 3.1. Let $N \in \mathbf{N}$. For a morphism $f: M \rightarrow M^{\prime}$ of abelian sheaves, we say that $f$ is $p^{N}$-injective (respectively, $p^{N}$-surjective) if its kernel (respectively, cokernel) is annihilated by $p^{N}$, and we say that $f$ is a $p^{N}$-isomorphism if it is $p^{N}$-injective and $p^{N}$-surjective. A morphism $f: M \rightarrow M^{\prime}$ in the derived category is a $p^{N}$-quasi-isomorphism if its cone has cohomology that is $p^{N}$-torsion. In particular, a $p^{N}$-acyclic complex is a complex whose cohomology groups are 


\section{ERTL AND W. NiziOŁ}

annihilated by $p^{N}$. We define a $p^{N}$-distinguished triangle as a triangle

$$
A \stackrel{f}{\rightarrow} B \stackrel{g}{\rightarrow} C \stackrel{h}{\rightarrow} A[1]
$$

together with a map to a distinguished triangle that is a $p^{N}$-quasi-isomorphism on each vertex. It follows that the associated long exact sequence of cohomology sheaves is $p^{2 N}$-acyclic. We note

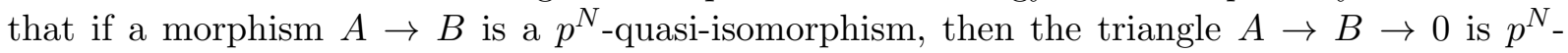
distinguished and, almost vice versa, if the triangle $A \rightarrow B \rightarrow 0$ is $p^{N}$-distinguished, then the morphism $A \rightarrow B$ is a $p^{3 N}$-quasi-isomorphism.

Now, we recall the following simple lemma.

Lemma 3.2 ([HK94, Lemma 4.18]). Let $C$ be an abelian category, $A$ an object of the derived category $D(C), S$ a finite subset of $\mathbf{Z}, m_{q}, q \in S$, integers, and assume $H^{q}(A)=0, q \notin S$, and that $H^{q}(A)$ is killed by $m_{q}$ for $q \in S$. Then $A$ is killed by $\prod_{q \in S} m_{q}$.

We will repeatedly use this lemma. Here is a typical example. Let $f: A \rightarrow B$ be a $p^{N}$-quasiisomorphism of complexes $A$ and $B$ concentrated in degrees $[0, m]$. Then there exists a morphism $g: B \rightarrow A$ such that $g f=p^{N(m) N}$, where $N(m)$ is a constant depending only on $m$; it is unique up to $p^{N(m) N}$; that is, if $g_{1}$ is another such morphism, then $p^{N(m) N} g=p^{N(m) N} g_{1}$. To see this, let $C$ be the cone of $f$. By assumption, $H^{i}(C)$ is $p^{N}$-torsion. Consider the exact sequence of Hom-groups in $D(C)$

$$
\operatorname{Hom}(C, A) \rightarrow \operatorname{Hom}(B, A) \stackrel{f^{*}}{\rightarrow} \operatorname{Hom}(A, A) \rightarrow \operatorname{Hom}(C[-1], A) .
$$

By Lemma 3.2, we have $p^{N(m) N} \operatorname{Hom}(C[-1], A)=0$. Hence, there exists a morphism $g$ as above. Since we also have $p^{N(m) N} \operatorname{Hom}(C, A)=0$, such a $g$ is $p^{N(m) N_{-}}$unique. We note that $g$ is a $p^{2 N_{-}}$ quasi-isomorphism. It also follows that $g(f g)=g p^{N(m) N}$. Using the exact sequence

$$
\operatorname{Hom}\left(C^{\prime}[-1], A\right) \rightarrow \operatorname{Hom}(A, A) \stackrel{g^{*}}{\rightarrow} \operatorname{Hom}(B, A) \rightarrow \operatorname{Hom}\left(C^{\prime}, A\right),
$$

where $C^{\prime}$ is the cone of $g$, we get $f\left(p^{2 N(m) N} g\right)=p^{3 N(m) N}$. Hence, if we put $h=p^{2 N(m) N} g$, we get $f h=p^{3 N(m) N}$ and $h f=p^{3 N(m) N}$.

Remark 3.3. It is clear to us that many of the denominators appearing in this paper can be improved upon with more careful bookkeeping. In particular, it is likely that the constants $N(d)$ depending on the dimension of the variety can be replaced by constants $N(r)$.

3.1.3 Cycle class map to syntomic cohomology. We state the following corollary of Theorem 2.5 .

Corollary 3.4. Let $X$ be a smooth variety over $K$. Then there exists a natural syntomic cycle class map

$$
\mathrm{cl}_{i, r}^{\mathrm{syn}}: H_{\mathrm{M}}^{i}\left(X, \mathbf{Q}_{p}(r)\right) \rightarrow H_{\mathrm{syn}}^{i}\left(X, \mathbf{Q}_{p}(r)\right),
$$

where the target group is the syntomic cohomology defined in [NN16]. This map is compatible with the étale cycle class map; that is, the following diagram commutes:

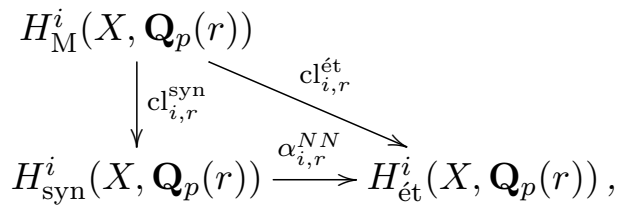




\section{Syntomic COHOMOLOGY AND p-ADIC MOTIVIC COHOMOLOGY}

where $\alpha_{i, r}^{N N}$ is the period map defined in [NN16, 4.2], where it is called $\rho_{\mathrm{syn}}$. Moreover, the cycle class map $\mathrm{cl}_{i, r}^{\mathrm{syn}}$ is an isomorphism for $i \leqslant r$.

Proof. For a semistable scheme $X$ over $\mathcal{O}_{K}$, consider the following diagram of sheaves on the Nisnevich site of $X$ :

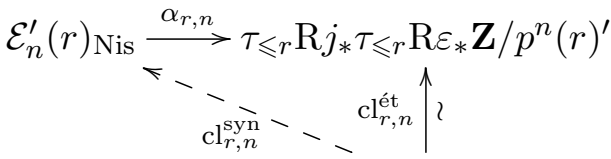

$$
\begin{aligned}
& \mathrm{R} j_{*} \mathbf{Z} / p^{n}(r)_{M}^{\prime} .
\end{aligned}
$$

The étale cycle class map $\mathrm{cl}_{r, n}^{\text {ét }}$ is a quasi-isomorphism by the Beilinson-Lichtenbaum conjecture (a corollary [SV00, GL01] of the Bloch-Kato conjecture proved by Voevodsky and Rost [Wei09]), by the quasi-isomorphism [GL00]

$$
\mathbf{Z} / p^{n}(r)_{M} \stackrel{\sim}{\rightarrow} \tau_{\leqslant r} \mathbf{R} \varepsilon_{*} \mathbf{Z} / p^{n}(r),
$$

and by the quasi-isomorphisms

$$
j_{*} \mathbf{Z} / p^{n}(r)_{M} \stackrel{\sim}{\rightarrow} \mathrm{R} j_{*} \mathbf{Z} / p^{n}(r)_{M} \quad \text { and } \quad \tau_{\leqslant r} \mathrm{R} j_{*} \mathbf{Z} / p^{n}(r)_{M} \stackrel{\sim}{\rightarrow} \mathrm{R} j_{*} \mathbf{Z} / p^{n}(r)_{M} .
$$

The period map $\alpha_{r, n}$ is a $p^{N r}$-quasi-isomorphism for a constant $N$ as described in Theorem 2.5. We claim that we can define compatible syntomic cycle class maps $\mathrm{cl}_{r, n}^{\mathrm{syn}}$ such that $\alpha_{r, n} \mathrm{cl}_{r, n}^{\mathrm{syn}}=p^{2 N r^{2}} \mathrm{cl}_{r, n}^{\text {ét }}$. To do this, take the cone $C_{n}$ of the map $h_{n}:=\left(\mathrm{cl}_{r, n}^{\text {ét }}\right)^{-1} \alpha_{r, n}$. It fits into the distinguished triangle

$$
\mathcal{E}_{n}^{\prime}(r)_{\mathrm{Nis}} \stackrel{h_{n}}{\longrightarrow} \mathrm{R} j_{*} \mathbf{Z} / p^{n}(r)_{M}^{\prime} \rightarrow C_{n},
$$

which yields the exact sequence of Hom-groups (in the derived category)

$$
\operatorname{Hom}\left(B_{n}, \mathcal{E}_{n}^{\prime}(r)_{\mathrm{Nis}}\right) \stackrel{h_{n}}{\longrightarrow} \operatorname{Hom}\left(B_{n}, B_{n}\right) \rightarrow \operatorname{Hom}\left(B_{n}, C_{n}\right),
$$

where we set $B_{n}:=\mathrm{R} j_{*} \mathbf{Z} / p^{n}(r)_{M}^{\prime}$.

Now, Lemma 3.2 applied to $C_{n}$ implies that $C_{n}$ is annihilated by $M:=p^{N r^{2}}$. Hence, so is $\operatorname{Hom}\left(B_{n}, C_{n}\right)$, and the exact sequence (3.1) gives that there exists a map $g_{n}: B_{n} \rightarrow \mathcal{E}_{n}^{\prime}(r)_{\text {Nis }}$ such that $h_{n} g_{n}=M$. We easily see that $\left(M g_{n}\right)$, for $n \geqslant 1$, is a morphism of pro-systems $\left\{B_{n}\right\} \rightarrow\left\{\mathcal{E}_{n}^{\prime}(r)_{\text {Nis }}\right\}$ such that $h_{n}\left(M g_{n}\right)=M^{2}$ and $\left(M g_{n}\right) h_{n}=M^{2}$ for $n \geqslant 1$. Set $\mathrm{cl}_{r, n}^{\text {syn }}:=M g_{n}$.

The above syntomic cycle class map $\mathrm{cl}_{r, n}^{\mathrm{syn}}$ induces the syntomic class map into syntomic cohomology

$$
\mathrm{cl}_{r, n}^{\mathrm{syn}}: \mathrm{R} j_{*} \mathbf{Z} / p^{n}(r)_{M} \stackrel{\mathrm{cl}_{r, n}^{\mathrm{syn}}}{\longrightarrow} \mathcal{E}_{n}^{\prime}(r)_{\mathrm{Nis}} \rightarrow \mathcal{S}_{n}^{\prime}(r)_{\mathrm{Nis}} \rightarrow \mathrm{R} \varepsilon_{*} \mathcal{S}_{n}^{\prime}(r)_{\text {ét }} .
$$

By construction, it is compatible with the étale cycle class map (via the map $\alpha_{r, n}$ and up to $p^{2 N r}$ ). Its rational version $\mathrm{cl}_{r, h}^{\mathrm{syn}} h$-sheafifies and gives the syntomic cycle class map

$$
\mathrm{cl}_{i, r}^{\mathrm{syn}}: H_{\mathrm{M}}^{i}\left(X, \mathbf{Q}_{p}(r)\right) \rightarrow H_{\mathrm{syn}}^{i}\left(X, \mathbf{Q}_{p}(r)\right), \quad \mathrm{cl}_{i, r}^{\mathrm{syn}}:=p^{-(2 N r-1) r} \mathrm{cl}_{i, r, h}^{\mathrm{syn}} .
$$

For the compatibility with the étale cycle class, it suffices to check that $\alpha_{i, r}^{N N}=p^{-r} \alpha_{i, r}$, but this was done by the second author in an upcoming paper.

We will describe how this $h$-sheafification works. Recall that the syntomic cohomology $H_{\text {syn }}^{i}\left(X, \mathbf{Q}_{p}(r)\right)$ is defined by $h$-sheafifying the (rational) Fontaine-Messing syntomic cohomology [NN16, 3.3]. More precisely, but simplifying enormously, the site $\mathcal{V} \operatorname{ar}_{K, h}$ of varieties over $K$ equipped with $h$-topology has a base consisting of proper semistable schemes $Y$ over $\mathcal{O}_{L}$, with $[L: K]<\infty$, such that $Y_{\operatorname{tr}} \rightarrow X$ is an $h$-map. It follows that to give a sheaf on $\mathcal{V}_{L, h}$, it suffices to describe its value on such $Y$. In particular, for the syntomic sheaf $\mathcal{S}^{\prime}(r)_{\mathbf{Q}}$ defined as 


\section{ERTL AND W. Niziǫ}

the $h$-sheafification of the presheaf sending $Y$ as above to $\mathrm{R} \Gamma_{\text {syn }}(Y, r)_{\mathbf{Q}}$, we set $H_{\text {syn }}^{i}\left(X, \mathbf{Q}_{p}(r)\right):=$ $H^{i}\left(X_{h}, \mathcal{S}^{\prime}(r)_{\mathbf{Q}}\right)$. We can also define the $h$-sheaf $\mathbf{Q}_{p}(r)_{M}$ by sending $Y$ to

$$
\left(\operatorname{holim}_{n} \mathbf{R} j_{*} \mathbf{Z} / p^{n}(r)_{M}(Y)\right)_{\mathbf{Q}}=\left(\operatorname{holim}_{n} \mathbf{Z} / p^{n}(r)_{M}\left(Y_{\mathrm{tr}}\right)\right)_{\mathbf{Q}} \text {. }
$$

Finally, we can $h$-sheafify all the other terms in (3.2) to obtain the map

$$
H_{\mathrm{M}, h}^{i}\left(X, \mathbf{Q}_{p}(r)\right):=H^{i}\left(X_{h}, \mathbf{Q}_{p}(r)_{M}\right) \rightarrow H_{\mathrm{syn}}^{i}\left(X, \mathbf{Q}_{p}(r)\right) .
$$

Composing it with the change of topology map $H_{\mathrm{M}, \mathrm{Nis}}^{i}\left(X, \mathbf{Q}_{p}(r)\right) \rightarrow H_{\mathrm{M}, h}^{i}\left(X, \mathbf{Q}_{p}(r)\right)$, we get the cycle class map $\mathrm{cl}_{r, h}^{\mathrm{syn}}$ we wanted.

The last claim of the corollary follows from the fact that both $\alpha_{i, r}^{N N}$ and clét $e_{i, r}$ are isomorphisms for $i \leqslant r$ by [NN16, Theorem A] and the Beilinson-Lichtenbaum conjecture, respectively.

\subsection{Syntomic cohomology and logarithmic de Rham-Witt cohomology}

We will show in this section that adding the logarithmic structure at the special fiber changes the syntomic cohomology by logarithmic de Rham-Witt cohomology, $W_{n} \Omega_{X_{0}, \log }^{*}$. Recall [Lor02] that in degree $q$, the latter is defined as the abelian subsheaf ${ }^{3}$ of $W_{n} \Omega_{X_{0}}^{q}$ generated locally by the symbols $d \log m_{1} \cdots \operatorname{dlog} m_{q}$ for $m_{1}, \ldots, m_{q}$ local sections of $M_{X_{0}}^{\mathrm{gp}}$. We note that if $x \in \mathcal{O}_{X_{0}}$, then $\operatorname{dlog} x=\operatorname{dlog}[x]=[x]^{-1} d([x])$.

Theorem 3.5. There exists a constant $N=N(p, d)$ or $N=N(p, d, e)$, depending on whether $K$ has enough roots of unity or not, such that for every $m \geqslant N$ and a semistable scheme $X$ over $\mathcal{O}_{K}$ with a smooth special fiber and of dimension $d$, we have the following natural $p^{m r}$-distinguished triangle of sheaves in the étale or Nisnevich topology of $X$, respectively:

$$
\mathcal{S}_{n}^{\prime}(r)_{X} \rightarrow \mathcal{S}_{n}^{\prime}(r)_{X^{\times}} \stackrel{\kappa_{m}}{\longrightarrow} W_{n} \Omega_{X_{0}, \mathrm{log}}^{r-1}[-r] \quad \text { or } \quad \mathcal{S}_{n}^{\prime}(r)_{X, \mathrm{Nis}} \quad \rightarrow \mathcal{S}_{n}^{\prime}(r)_{X \times, \mathrm{Nis}} \stackrel{\kappa_{m}}{\longrightarrow} W_{n} \Omega_{X_{0}, \log }^{r-1}[-r] .
$$

Here we write $X^{\times}$for the scheme $X$ with added log-structure coming from the special fiber. These triangles are compatible for different choices of $m$.

Proof. We will find such a constant $N$ and the triangles corresponding to it. For $m \geqslant N$, we simply set $\kappa_{m}:=p^{(m-N) r} \kappa_{N}$.

After setting up the local coordinates, we do, as an example, computations in dimension 0, where it becomes clear how to define the map to logarithmic de Rham-Witt differentials. Then we lift these computations to higher dimensions and globalize.

Step 1: Choice of local coordinates. To construct the first distinguished triangle, we start with local computations. Let $d$ be a positive integer satisfying $a \leqslant d$. Let

$$
R_{K}^{0}:=\mathcal{O}_{K}\left\{X_{1}^{ \pm 1}, \ldots, X_{a}^{ \pm 1}, X_{a+1}, \ldots, X_{d}\right\}
$$

be the $p$-adic completion of $\mathcal{O}_{K}\left[X_{1}^{ \pm 1}, \ldots, X_{a}^{ \pm 1}, X_{a+1}, \ldots, X_{d}\right]$. Let $R$ be the $p$-adic completion of an étale algebra over $R_{K}^{0}$. Let $R_{T}^{0}$ be the $(p, T)$-adic completion of $W(k)\left[T, X_{1}^{ \pm 1}, \ldots, X_{a}^{ \pm 1}\right.$, $\left.X_{a+1}, \ldots, X_{d}\right]$; take the map $R_{T}^{0} \rightarrow R_{K}^{0}, T \mapsto \varpi$, and take the (formally) étale lifting $R_{T}$ of $R$ to $R_{T}^{0}$. Let $S_{R}$ be the $p$-adically complete PD-envelope of $R$ in $R_{T}$ equipped with the PD-filtration $F^{r} S_{R}$. We will write $S_{K}:=S_{\mathcal{O}_{K}}$. We have $S_{R}=R_{T} \widehat{\otimes}_{W(k)\{T\}} S_{K}$ with filtration $F^{r} S_{R}:=R_{T} \widehat{\otimes}_{W(k)\{T\}} F^{r} S_{K}$. Let $R^{0}:=W(k)\left\{X_{1}^{ \pm 1}, \ldots, X_{a}^{ \pm 1}, X_{a+1}, \ldots, X_{d}\right\}$, and let $R_{T, 0}:=$ $R_{T} / T$.

\footnotetext{
${ }^{3}$ We will use étale or Nisnevich topology.
} 


\section{SyNTOMiC COHOMOLOGY AND p-ADIC MOTIVIC COHOMOLOGY}

We have the following diagrams of maps (the right diagram is obtained by reducing the rings modulo $T$ ):
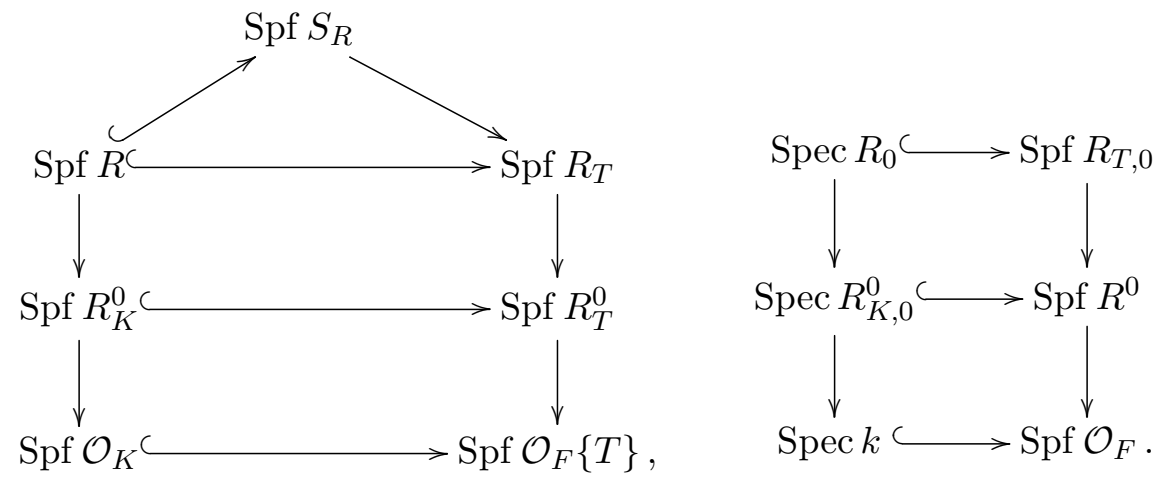

Equip $R^{0}$ with a Frobenius morphism $\varphi_{R^{0}}: X_{i}^{ \pm 1} \mapsto X_{i}^{ \pm p}$. Equip $R_{T}^{0}$ with a Frobenius morphism $\varphi_{R_{T}^{0}}$ compatible with $\varphi_{S_{K}}\left(T \mapsto T^{p}\right)$ and with $\varphi_{R^{0}}$, and equip $R_{T}$ with a Frobenius morphism $\varphi_{R_{T}}$ compatible with $\varphi_{R_{T}^{0}}$. We will simply write $\varphi$ for the Frobenius morphism if the domain of action is understood. The natural $\log$-structure on $\operatorname{Spf} R$ is given by the special fiber and the divisor at infinity, $X_{a+1} \cdots X_{d}=0$. It is described by the monoid $M=\mathbf{N}^{d-a+1}$ and the map defined on the generators by $e_{0} \mapsto \varpi$ and $e_{i} \mapsto X_{i}$ for $a+1 \leqslant i \leqslant d$.

Set $\Omega_{S_{R}}:=S_{R} \otimes_{R_{T}} \Omega_{R_{T}}$. For $r \in \mathbf{N}$, we filter the de Rham complex $\Omega_{S_{R}}^{\bullet}$ by subcomplexes

$$
F^{r} \Omega_{S_{R}}^{\bullet}:=F^{r} S_{R} \rightarrow F^{r-1} S_{R} \otimes_{R_{T}} \Omega_{R_{T}} \rightarrow F^{r-2} S_{R} \otimes_{R_{T}} \Omega_{R_{T}}^{2} \rightarrow \cdots .
$$

We define the syntomic complex of $R$ as

$$
S(R, r):=\text { Cone }\left(F^{r} \Omega_{S_{R}}^{\bullet} \stackrel{p^{r}-\varphi}{\longrightarrow} \Omega_{S_{R}}^{\bullet}\right)[-1] .
$$

Set $\Omega_{S_{R}^{\times}}:=S_{R} \otimes_{R_{T}} \Omega_{R_{T}^{\times}}$, where $R_{T}^{\times}$is the ring $R_{T}$ with $\log$-structure induced by $T$. We define the log-syntomic complex of $R$ as

$$
S\left(R^{\times}, r\right):=\text { Cone }\left(F^{r} \Omega_{S_{R}^{\times}}^{\bullet} \stackrel{p^{r}-\varphi}{\longrightarrow} \Omega_{S_{R}^{\times}}^{\bullet}\right)[-1] .
$$

For $n \in \mathbf{N}$, we define the syntomic and log-syntomic complexes modulo $p^{n}$ as $S(R, r)_{n}:=$ $S(R, r) \otimes_{\mathbf{Z}} \mathbf{Z} / p^{n}$ and $S\left(R^{\times}, r\right)_{n}:=S\left(R^{\times}, r\right) \otimes_{\mathbf{Z}} \mathbf{Z} / p^{n}$, respectively. In the case when $\widehat{R}$ is the $p$-adic completion of an étale algebra $R$ over $\mathcal{O}_{K}\left[X_{1}^{ \pm 1}, \ldots, X_{d}^{ \pm 1}, X_{a+1}, \ldots, X_{d}\right]$, we have

$$
\begin{aligned}
S_{n}^{\prime}(r)_{R} & =S(\widehat{R}, r)_{n}, \quad S_{n}^{\prime}(r)_{R^{\times}}=S\left(\widehat{R}^{\times}, r\right)_{n}, \\
\operatorname{holim}_{n} S_{n}^{\prime}(r)_{R} & =S(\widehat{R}, r), \quad \operatorname{holim}_{n} S_{n}^{\prime}(r)_{R^{\times}}=S\left(\widehat{R}^{\times}, r\right) .
\end{aligned}
$$

We would like to separate the arithmetic and geometric variables. Specifically, we remove the differentials connected with the variable $T$ by setting $\Omega_{S_{R}^{\prime}}:=S_{R} \otimes_{R^{0}} \Omega_{R^{0}}$. Since $\Omega_{W(k)[T]}=$ $W(k)[T] d T$, we can dispose of this module of differentials by writing $d f$ as $\partial f d T$, and we can rewrite the above syntomic complex as the following homotopy limit:

$$
S(R, r)=\left[\begin{array}{cc}
F^{r} \Omega_{S_{R}^{\prime}} \stackrel{p^{r}-p^{\bullet} \varphi \bullet}{\longrightarrow} & \Omega_{S_{R}^{\prime}} \\
\downarrow & \downarrow \\
\partial & \partial \\
F^{r-1} \Omega_{S_{R}^{\prime}} \stackrel{p^{r}-p^{\bullet+1} T^{p-1} \varphi \bullet}{\longrightarrow} \Omega_{S_{R}^{\prime}}^{\bullet}
\end{array}\right] .
$$

Here the map $\varphi_{\bullet}: \Omega_{S_{R}^{\prime}}^{\bullet} \rightarrow \Omega_{S_{R}^{\prime}}^{\bullet}$ sends $\omega \in \Omega_{S_{R}^{\prime}}^{k}$ to $\left(\varphi / p^{k}\right)(\omega)$. By adding logarithmic differentials 


\section{ERTL AND W. NiziOŁ}

$d T / T$ along the special fiber, we get the following log-syntomic complex:

$$
S\left(R^{\times}, r\right)=\left[\begin{array}{ccc}
F^{r} \Omega_{S_{R}^{\prime}}^{\bullet} & p^{r}-p^{\bullet} \varphi \bullet & \Omega_{S_{R}^{\prime}}^{\bullet} \\
\downarrow & & \mid T \partial \\
\downarrow & & \downarrow \\
F^{r-1} \Omega_{S_{R}^{\prime}}^{\bullet} & p^{r}-p^{\bullet+1} \varphi \bullet & \Omega_{S_{R}^{\prime}}^{\bullet}
\end{array}\right] .
$$

In this language, the natural map $S(R, r) \rightarrow S\left(R^{\times}, r\right)$ is given by multiplication by $T$ on the bottom row.

Step 2: Dimension 0. For $R=\mathcal{O}_{K}$, we obtain the following proposition.

Proposition 3.6. Let $n \geqslant 1$. We have a compatible family of $p^{48}$-distinguished triangles of sheaves in the étale topology of Spec $k$,

$$
S\left(\mathcal{O}_{K}, 1\right)_{n} \rightarrow S\left(\mathcal{O}_{K}^{\times}, 1\right)_{n} \rightarrow \mathbf{Z} / p^{n}[-1]
$$

For $r \neq 1$, the natural map $S\left(\mathcal{O}_{K}, r\right)_{n} \rightarrow S\left(\mathcal{O}_{K}^{\times}, r\right)_{n}$ is a $p^{48 r}$-quasi-isomorphism.

Proof. Using the above homotopy limit presentations, we write down two syntomic complexes

$$
\begin{aligned}
& S\left(\mathcal{O}_{K}, r\right): F^{r} S_{K} \stackrel{\left(\partial, p^{r}-\varphi\right)}{\longrightarrow} F^{r-1} S_{K} \oplus S_{K} \stackrel{-\left(p^{r}-p T^{p-1} \varphi\right)+\partial}{\longrightarrow} S_{K}, \\
& S\left(\mathcal{O}_{K}^{\times}, r\right): F^{r} S_{K} \stackrel{\left(T \partial, p^{r}-\varphi\right)}{\longrightarrow} F^{r-1} S_{K} \oplus S_{K} \stackrel{-\left(p^{r}-p \varphi\right)+T \partial}{\longrightarrow} S_{K} .
\end{aligned}
$$

The natural map $S\left(\mathcal{O}_{K}, r\right) \rightarrow S\left(\mathcal{O}_{K}^{\times}, r\right)$ is given by the multiplication by $T$ on $F^{r-1} S_{K}$ and the last $S_{K}$.

Let $S_{K}^{[1]}$ be the $p$-adic completion of $W(k)\left[T, T^{i} / p^{[i / e]}, i \in \mathbf{N}\right]$. Elements of $S_{K}^{[1]}$ can be written uniquely in the form $\sum_{i \in \mathbf{N}} a_{i} T^{i} / p^{[i / e]}$, where $a_{i} \in W(k)$ with $a_{i} \rightarrow 0$ as $i \rightarrow \infty$. They form the ring of analytic functions over $F$ with integral values on the disk $v_{p}(T) \geqslant 1 / e$. We have $S_{K} \subset S_{K}^{[1]}$. The formulas (3.4) and (3.5) make sense with $S_{K}$ replaced by $S_{K}^{[1]}$. We call the resulting complexes the syntomic complexes of $\mathcal{O}_{K}^{[1]}$ and denote them by $S\left(\mathcal{O}_{K}^{[1]}, r\right)$ and $S_{\log }\left(\mathcal{O}_{K}^{[1]}, r\right)$, respectively. The natural maps

$$
S\left(\mathcal{O}_{K}, r\right) \rightarrow S\left(\mathcal{O}_{K}^{[1]}, r\right), \quad S\left(\mathcal{O}_{K}^{\times}, r\right) \rightarrow S_{\log }\left(\mathcal{O}_{K}^{[1]}, r\right)
$$

are $p^{6 r}$-quasi-isomorphisms. In the case of the second map, this is [CN17, Proposition 3.3]. A simple corollary of [CN17, Lemma 3.2] states that the map $p^{s}-\varphi$, for $s=r, r-1$, induces a $p^{s+r}$ isomorphism $F^{r} \Omega_{S_{K}^{[1]}}^{i} / F^{r} \Omega_{S_{K}}^{i} \simeq \Omega_{S_{K}^{[1]}}^{i} / \Omega_{S_{K}}^{i}$. This lemma holds also for the map $p^{s}-T^{p-1} \varphi$, with basically the same proof, which implies that the first map in (3.8) is a $p^{6 r}$-quasi-isomorphism as well.

The residue map $\operatorname{res}_{T}: \Omega_{\log , S_{K}^{[1]}}^{1} \rightarrow \mathcal{O}_{F}$ induces the following sequence of complexes:

$$
0 \rightarrow S\left(\mathcal{O}_{K}^{[1]}, r\right) \rightarrow S_{\log }\left(\mathcal{O}_{K}^{[1]}, r\right) \stackrel{\operatorname{res}_{T}}{\longrightarrow}\left(0 \rightarrow \mathcal{O}_{F} \stackrel{-\left(p^{r}-p \varphi\right)}{\longrightarrow} \mathcal{O}_{F}\right) \rightarrow 0
$$

The above sequence is $p$-exact because $F^{s} S_{K}^{[1]}=p^{-s} E^{s} S_{K}^{[1]}$, for $E$ the minimal polynomial of $\varpi$ over $F$, which implies that $F^{s} \Omega_{\log , S_{K}^{[1]}} / F^{s} \Omega_{S_{K}^{1]}}^{[1]} \cong S_{K}^{[1]} / T S_{K}^{[1]}$ and $S_{K}^{[1]} / T S_{K}^{[1]}=\mathcal{O}_{F} \oplus M$, where $M$ is $p$-torsion.

Modulo $p^{n}$, we have $\mathcal{O}_{F, n}=W_{n}(k)$ and the exact sequence in the étale topology of Spec $k$

$$
0 \rightarrow \mathbf{Z} / p^{n} \rightarrow \mathcal{O}_{F, n} \stackrel{1-\varphi}{\longrightarrow} \mathcal{O}_{F, n} \rightarrow 0 \text {. }
$$




\section{SyNTOMiC COHOMOLOGY AND p-ADIC MOTIVIC COHOMOLOGY}

For $r=0$, the map $p^{r}-p \varphi: \mathcal{O}_{F, n} \rightarrow \mathcal{O}_{F, n}$ is an isomorphism since $1-p \varphi$ is invertible. For $r>1$, the map $p^{r-1}-\varphi: \mathcal{O}_{F, n} \rightarrow \mathcal{O}_{F, n}$ is an isomorphism as well since both $\varphi$ and $p^{r-1} \varphi^{-1}-1$ are invertible. Our proposition is now proved using Section 3.1.2.

Step 3: Local computations in higher dimensions. The computations in the above example generalize to any ring $R$.

Lemma 3.7. There exist a constant $N=N(p, d)$ or $N=N(p, d, e)$, depending on whether $K$ has enough roots of unity or not, and a natural $p^{N r}$-distinguished triangle in the étale topology of $\operatorname{Spec} R_{0}$,

$$
S(R, r)_{n} \rightarrow S\left(R^{\times}, r\right)_{n} \stackrel{\kappa_{N}}{\longrightarrow} W_{n} \Omega_{R_{0}, \log }^{r-1}[-r] .
$$

Proof. We claim that the triangle

$$
S(R, r) \rightarrow S_{\log }(R, r) \stackrel{\operatorname{res}_{T}}{\longrightarrow}\left[\Omega_{R_{T, 0}}^{\bullet} \stackrel{p^{r}-p^{\bullet+1} \varphi \bullet}{\longrightarrow} \Omega_{R_{T, 0}}^{\bullet}\right][-1]
$$

is $p^{N r}$-distinguished for $N$ as in the theorem. We note that the complex $\Omega_{R_{T, 0}}^{\bullet}$ computes the crystalline cohomology of $R_{0}$ over $W(k)$. To prove the claim, we can assume $r>0$ since it is clear for $r=0$. Set $S_{R}^{[1]}:=R_{T} \widehat{\otimes}_{W(k)[T]} S_{K}^{[1]}$ with the induced Frobenius morphism and filtration. Define syntomic cohomology complexes $S\left(R^{[1]}, r\right)$ and $S_{\log }\left(R^{[1]}, r\right)$ by formulas (3.4) and (3.5), replacing $S_{R}$ with $S_{R}^{[1]}$. Just as above, in dimension 0, we can pass from the syntomic cohomology of $R$ to the syntomic cohomology of $R^{[1]}$ via a $p^{6 r}$-quasi-isomorphism. It now suffices to show that the triangle

$$
S\left(R^{[1]}, r\right) \rightarrow S_{\log }\left(R^{[1]}, r\right) \stackrel{\operatorname{res}_{T}}{\longrightarrow}\left[\Omega_{R_{T, 0}}^{\bullet} \stackrel{p^{r}-p^{\bullet+1} \varphi \bullet}{\longrightarrow} \Omega_{R_{T, 0}}\right][-1]
$$

is $p^{N r}$-distinguished for $N$ as in the theorem.

Using the homotopy limit presentations (3.6) and (3.7), we get the exact sequence

$$
0 \rightarrow S\left(R^{[1]}, r\right) \rightarrow S_{\log }\left(R^{[1]}, r\right) \rightarrow\left[F^{r} \Omega_{S_{R}^{[1],}}^{[} / T \stackrel{p^{r}-p^{\bullet+1} \varphi \bullet}{\longrightarrow} \Omega_{S_{R}^{\bullet 1], /}} / T\right][-1] \rightarrow 0,
$$

where $\Omega_{S_{R}^{[1], \prime}}=S_{R}^{[1]} \otimes_{R^{0}} \Omega_{R^{0}}=S_{K}^{[1]} \widehat{\otimes}_{W(k)\{T\}} R_{T} \otimes_{R^{0}} \Omega_{R^{0}}$. By Section 3.1.2, it suffices to show that the map

$$
\operatorname{res}_{T}:\left[F^{r} \Omega_{S_{R}^{[1],}}^{\bullet} / T \stackrel{p^{r}-p^{\bullet+1} \varphi \bullet}{\longrightarrow} \Omega_{S_{R}^{[1],,}}^{\bullet} / T\right] \rightarrow\left[\Omega_{R_{T, 0}}^{\bullet} \stackrel{p^{r}-p^{\bullet+1} \varphi \bullet}{\longrightarrow} \Omega_{R_{T, 0}}^{\bullet}\right]
$$

is a $p^{2}$-quasi-isomorphism. The complex on the left can be simplified. We have (see the proof of Proposition 3.6)

$$
\begin{aligned}
F^{s} \Omega_{S_{R}^{[1,,}} / T & =\left(F^{s} S_{R}^{[1]} / T\right) \otimes_{R^{0}} \Omega_{R^{0}}=\left(F^{s} S_{K}^{[1]} / T\right) \widehat{\otimes}_{W(k)} R_{T, 0} \otimes_{R^{0}} \Omega_{R^{0}} \\
& \simeq\left(\left(\widetilde{E}^{s} / p^{s}\right) S_{K}^{[1]}\right) / T \widehat{\otimes}_{W(k)} R_{T, 0} \otimes_{R^{0}} \Omega_{R^{0}} \simeq\left(\mathcal{O}_{F} \oplus M\right) \widehat{\otimes}_{W(k)} R_{T, 0} \otimes_{R^{0}} \Omega_{R^{0}},
\end{aligned}
$$

for a $p$-torsion module $M$. Here $\widetilde{E}$ is a twist of $E=a_{e} T^{e}+\cdots+a_{1} T+a_{0}$, the minimal polynomial of $\varpi$ over $\mathcal{O}_{F}$. Note that since $E$ is Eisenstein, $u=a_{0} p^{-1}$ is a unit. We set $\widetilde{E}:=u^{-1} E$; its constant coefficient is equal to $p$. Hence, the residue map

$$
\begin{aligned}
\operatorname{res}_{T}: F^{r} \Omega_{S_{R}}^{\bullet[1,,} / T & =\left(F^{r} \Omega_{S_{R}^{[1],}}^{0} / T \rightarrow F^{r-1} \Omega_{S_{R}^{[1],}}^{1} / T \rightarrow F^{r-2} \Omega_{S_{R}^{[1],}}^{2} / T \rightarrow \cdots\right) \\
& \rightarrow\left(\Omega_{R_{T, 0}^{0}}^{0} \stackrel{d_{0}}{\rightarrow} \Omega_{R_{T, 0}}^{1} \stackrel{d_{1}}{\rightarrow} \Omega_{R_{T, 0}^{2}}^{2} \stackrel{d_{2}}{\rightarrow} \cdots\right)
\end{aligned}
$$

is a $p$-quasi-isomorphism. It follows that the map (3.11) is a $p^{2}$-quasi-isomorphism, as wanted. 


\section{ERTL AND W. NiZIOE}

Set $S:=R_{T, 0}$. We claim that there exists a $p^{N r}$-quasi-isomorphism, for $N$ as in the theorem, on the étale site of $\operatorname{Spec} R_{0}$,

$$
\left[\Omega_{S, n}^{\bullet} \stackrel{p^{r}-p^{\bullet+1} \varphi \bullet}{\longrightarrow} \Omega_{S, n}^{\bullet} \underset{\leftarrow}{\leftarrow} W_{n} \Omega_{R_{0}, \log }^{r-1}[-r+1] .\right.
$$

Indeed, for $r=0$, the complex $\left[\Omega_{S, n}^{\bullet} \stackrel{1-p^{\bullet+1} \varphi \bullet}{\longrightarrow} \Omega_{S, n}^{\bullet}\right]$ is acyclic because the map $1-p^{\bullet+1}$ is invertible. Thus, assume $r \geqslant 1$ and take $s=r-1$. Set

$$
\operatorname{HK}(S, s)_{n}:=\left[\Omega_{S, n}^{\bullet} \stackrel{p^{s}-p^{\bullet} \varphi}{\longrightarrow} \Omega_{S, n}^{\bullet}\right] .
$$

This complex is $p^{2}$-quasi-isomorphic to the complex $\left[\Omega_{S, n}^{\bullet} \stackrel{p^{r}-p^{\bullet+1} \varphi \bullet}{\longrightarrow} \Omega_{S, n}^{\bullet}\right]$. Using the global Frobenius lift on $S$, we get the following commutative diagram:

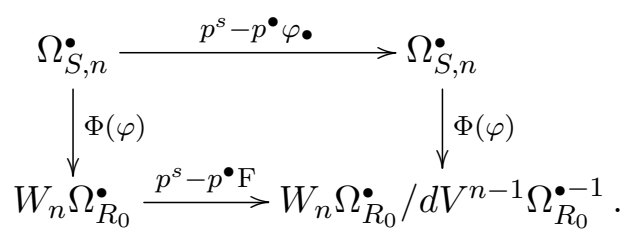

We note here that the de Rham-Witt Frobenius morphism F: $W_{n+1} \Omega_{R_{0}}^{\bullet} \rightarrow W_{n} \Omega_{R_{0}}^{\bullet}$ restricts to a morphism

$$
\mathrm{F}: \mathrm{Fil}^{n} W_{n+1} \Omega_{R_{0}}^{\bullet}=V^{n} \Omega_{R_{0}}^{\bullet}+d V^{n} \Omega_{R_{0}}^{\bullet-1} \rightarrow d V^{n-1} \Omega_{R_{0}}^{\bullet-1} .
$$

Hence $\mathrm{F}$ factors as in the above diagram. Moreover, since $p d V^{n-1} \Omega_{R_{0}}^{*}=0$, we get the induced $\operatorname{map} p \mathrm{~F}: W_{n} \Omega_{R_{0}}^{\bullet} \rightarrow W_{n} \Omega_{R_{0}}^{\bullet}$.

The first vertical arrow in the above diagram is a quasi-isomorphism. The second one is a $p$ quasi-isomorphism since $p d V^{n-1} \Omega_{R_{0}}^{*}=0$. Hence, the complex $\operatorname{HK}(S, s)_{n}$ is $p^{2}$-quasi-isomorphic to the complex $\left[W_{n} \Omega_{R_{0}}^{\bullet} \stackrel{p^{s}-p^{\bullet} \mathrm{F}}{\longrightarrow} W_{n} \Omega_{R_{0}}^{\bullet} / d V^{n-1} \Omega_{R_{0}}^{\bullet-1}\right.$. We list the following properties of the latter complex:

(1) For $t>s$, the map $1-p^{t-s} \mathrm{~F}: W_{n} \Omega_{R_{0}}^{t} \rightarrow W_{n} \Omega_{R_{0}}^{t}$ is an isomorphism (since $1-p^{t-s} \mathrm{~F}$ is invertible).

(2) For $t<s$, the map

$$
p^{s-t}-\mathrm{F}: W_{n} \Omega_{R_{0}}^{t} \rightarrow W_{n} \Omega_{R_{0}}^{t} / d V^{n-1} \Omega_{R_{0}}^{t-1}
$$

is a $p$-isomorphism. Indeed, for the $p$-surjectivity it suffices to note that $\left(p^{s-t}-\mathrm{F}\right)(V \alpha)=$ $p^{s-t} V \alpha-p \alpha$ for $\alpha \in W_{n} \Omega_{R_{0}}^{t} / d V^{n-1} \Omega_{R_{0}}^{t-1}$ and $t \leqslant s-1$. For the $p$-injectivity, we note that if $\left(p^{s-t}-\mathrm{F}\right)(\alpha)=0$ for $\alpha \in W_{n} \Omega_{R_{0}}^{t}$, then $V\left(p^{s-t}-\mathrm{F}\right)(\alpha)=p^{s-t} V \alpha-p \alpha=0$. Hence, $p^{s-t-1} V \alpha=\alpha$, which implies $p^{n(s-t-1)} V^{n} \alpha=\alpha$. Hence, $\alpha=0$.

(3) There is an exact sequence

$$
0 \rightarrow W_{n} \Omega_{R_{0}, \log }^{s} \rightarrow W_{n} \Omega_{R_{0}}^{s} \stackrel{1-\mathrm{F}}{\longrightarrow} W_{n} \Omega_{R_{0}}^{s} / d V^{n-1} \Omega_{R_{0}}^{s-1} \rightarrow 0
$$

in the étale topology of Spec $R_{0}$ [CSS83, Lemma 1.2], [Lor02, Proposition 2.13]. In the Nisnevich topology it is still exact on the left and in the middle.

Consider the following sequence of complexes on the étale site:

$$
0 \rightarrow W_{n} \Omega_{R_{0}, \log }^{s}[-s] \rightarrow W_{n} \Omega_{R_{0}}^{\bullet} \stackrel{p^{s}-p^{\bullet} \mathrm{F}}{\longrightarrow} W_{n} \Omega_{R_{0}}^{\bullet} / d V^{n-1} \Omega_{R_{0}}^{\bullet-1} \rightarrow 0 .
$$

By point (3) above it is $p^{s}$-exact in degree $s$. By point (2), it is $p^{s}$-exact in degrees less than $s$. In degrees $s+i>s$, it becomes the sequence

$$
0 \rightarrow W_{n} \Omega_{R_{0}}^{s+i} \stackrel{p^{s}-p^{s+i} \mathrm{~F}}{\longrightarrow} W_{n} \Omega_{R_{0}}^{s+i} / d V^{n-1} \Omega_{R_{0}}^{s+i-1} \rightarrow 0 .
$$




\section{SyNTOMiC COHOMOLOGY AND p-ADIC MOTIVIC COHOMOLOGY}

By point (1), it is $p^{s}$-exact on the right; by the same point and the fact that $p d V^{n-1} \Omega_{R_{0}}^{*}=0$, it is also $p^{s}$-exact on the left. Thus the natural map

$$
W_{n} \Omega_{R_{0}, \log }^{s}[-s] \rightarrow\left[W_{n} \Omega_{R_{0}}^{\bullet} \stackrel{p^{s}-p^{\bullet} \mathrm{F}}{\longrightarrow} W_{n} \Omega_{R_{0}}^{\bullet} / d V^{n-1} \Omega_{R_{0}}^{\bullet-1}\right]
$$

is a $p^{4 s}$-quasi-isomorphism in the étale topology of $\operatorname{Spec} R_{0}$, as wanted.

We obtain the quasi-isomorphism (3.12) by appealing to Section 3.1.2. Then, using the same section, we get our lemma.

Step 4: Globalization; the first triangle. The above local computations can be globalized in the case of the first triangle in the theorem in the following way. We note that we have actually proved above that we have the following $p^{4 s}$-quasi-isomorphisms of sheaves on the étale site of $X_{0}$ :

$$
W_{n} \Omega_{X_{0}, \log }^{s}[-s] \stackrel{\beta_{1}}{\longrightarrow}\left[W_{n} \Omega_{X_{0}}^{p^{s}-p^{\bullet}} \stackrel{F}{\longrightarrow} W_{n} \Omega_{X_{0}}^{\bullet} / d V^{n-1} \Omega_{X_{0}}^{\bullet-1}\right] \stackrel{\beta_{2}}{\longleftarrow}\left[\mathcal{A}_{\mathrm{cr}, n} \stackrel{p^{s}-\varphi}{\longrightarrow} \mathcal{A}_{\mathrm{cr}, n}\right],
$$

where $\mathcal{A}_{\mathrm{cr}, n}$ is the sheaf $\left(U \rightarrow X_{0}\right) \mapsto \mathrm{R} \Gamma_{\mathrm{cr}}\left(U / W_{n}(k)\right)$. The notation is slightly abusive here, but we hope that this will not lead to confusion. The second $p$-quasi-isomorphism follows from Illusie's comparison quasi-isomorphism $\mathcal{A}_{\mathrm{cr}, n} \stackrel{\sim}{\rightarrow} W_{n} \Omega_{X_{0}}$ [Ill79, Section II.1]. By Section 3.1.2, for $N=N(d)$, there exists a $p^{N s}$-section $\gamma_{1}$ of the map $\beta_{1}$, that is, a map $\gamma_{1}$ such that $\gamma_{1} \beta_{1}=p^{N s}$ and $\beta_{1} \gamma_{1}=p^{N s}$. It thus suffices to construct a map

$$
\mathcal{S}_{n}^{\prime}(r)_{X^{\times}} \rightarrow\left[\mathcal{A}_{\mathrm{cr}, n} \stackrel{p^{r}-p \varphi}{\longrightarrow} \mathcal{A}_{\mathrm{cr}, n}\right][-1]
$$

and show that the triangle

$$
\mathcal{S}_{n}^{\prime}(r)_{X} \rightarrow \mathcal{S}_{n}^{\prime}(r)_{X^{\times}} \rightarrow\left[\mathcal{A}_{\mathrm{cr}, n} \stackrel{p^{r}-p \varphi}{\longrightarrow} \mathcal{A}_{\mathrm{cr}, n}\right][-1]
$$

is $p^{N r}$-distinguished for $N$ as in the theorem.

For this, consider the following two diagrams of compatible coordinate systems (localize on $X$ if necessary to get $X=\operatorname{Spec} A$ ):
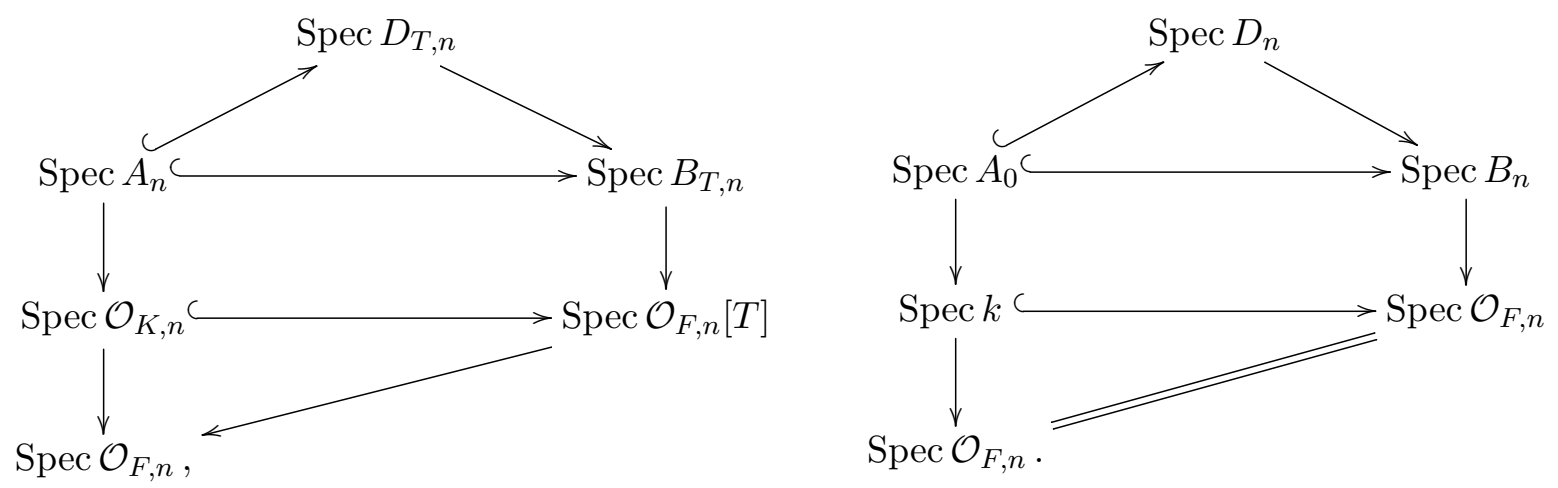

Here $B_{T, n}$ is log-smooth over $\operatorname{Spec} \mathcal{O}_{F, n}[T]$, where the latter scheme is equipped with the logstructure associated to $T$, and the hooked arrows are exact closed embeddings. The right diagram is obtained by "reducing" the left diagram "modulo $T$." It follows that the residue map $\operatorname{res}_{T}: \Omega_{B_{T, n}^{\times}} \rightarrow \mathcal{O}_{B_{n}}$ induces a map $\operatorname{res}_{T}: \Omega_{D_{T, n}^{\times}}^{\bullet} \rightarrow \Omega_{D_{n}}^{\bullet-1}$ (we note that the Frobenius morphism $\varphi$ on the domain is compatible with $p \varphi$ on the target) and that the sequence

$$
0 \rightarrow \Omega_{D_{T, n}}^{\bullet} \rightarrow \Omega_{D_{T, n}^{\times}}^{\bullet} \stackrel{\operatorname{res}_{T}}{\longrightarrow} \Omega_{D_{n}}^{\bullet-1} \rightarrow 0
$$

is exact. These constructions glue in the usual way, and we obtain a map $\operatorname{res}_{T}: \mathcal{J}_{X_{n}^{\times}}^{[r]} \rightarrow \mathcal{A}_{\mathrm{cr}, n}[-1]$ 


\section{ERTL AND W. NiziOŁ}

and a sequence of complexes of sheaves on the étale site of $X_{0}$

$$
\mathcal{J}_{X_{n}}^{[r]} \rightarrow \mathcal{J}_{X_{n}^{\times}}^{[r]} \stackrel{\operatorname{res}_{T}}{\longrightarrow} \mathcal{A}_{\mathrm{cr}, n}[-1]
$$

where we write $\mathcal{J}_{X_{n}^{*}}^{[r]}$ for the sheaf $\left(U \rightarrow X_{n}\right) \mapsto \mathrm{R} \Gamma_{\mathrm{cr}}\left(U, \mathcal{J}_{X_{n}^{*}}^{[r]}\right)$. Hence, we obtain a sequence

$$
\mathcal{S}_{n}^{\prime}(r)_{X} \rightarrow \mathcal{S}_{n}^{\prime}(r)_{X^{\times}} \stackrel{\operatorname{res}_{T}}{\longrightarrow}\left[\mathcal{A}_{\mathrm{cr}, n} \stackrel{p^{r}-p \varphi}{\longrightarrow} \mathcal{A}_{\mathrm{cr}, n}\right][-1] .
$$

It is a $p^{N r}$-distinguished triangle for $N$ as in the theorem: this can be checked locally, where we can pass to the more convenient coordinate system from (3.3) and use the computations we have done in the proof of Proposition 3.6. Define the map $\kappa_{N}$ in our theorem as the composition $\gamma_{1} \beta_{2} \operatorname{res}_{T}$ for a fixed choice of such an $N$. This concludes the construction of the first distinguished triangle of our theorem.

Step 5: Symbols and the map $\kappa_{N}$. Before continuing, we need to understand the relation between syntomic symbols and the map $\kappa_{N}$ defined above.

Lemma 3.8. (1) For $f_{i} \in \mathcal{O}_{X}^{*}(X)$, for $1 \leqslant i \leqslant r$, we have

$$
\kappa_{N}\left(\left\{f_{1}, \ldots, f_{r}\right\}\right)=0, \quad \kappa_{N}\left(\left\{f_{1}, \ldots, f_{r-1}, \varpi\right\}\right)=p^{N} \operatorname{dlog}\left[\bar{f}_{1}\right] \cdots \operatorname{dlog}\left[\bar{f}_{r-1}\right],
$$

where $\bar{f}_{i}$ is the reduction of $f_{i}$ to $\mathcal{O}_{X_{0}}^{*}$.

(2) For $f_{i} \in M_{X}^{\mathrm{gp}}(X)$, for $1 \leqslant i \leqslant r$, we have

$$
\kappa_{N}\left(\left\{f_{1}, \ldots, f_{r}\right\}\right)=0, \quad \kappa_{N}\left(\left\{f_{1}, \ldots, f_{r-1}, \varpi\right\}\right)=p^{N} \operatorname{dlog} \bar{f}_{1} \cdots \operatorname{dlog} \bar{f}_{r-1},
$$

where $\bar{f}_{i}$ is the reduction of $f_{i}$ to $M_{X_{0}}^{\mathrm{gp}}$.

Proof. It is enough to argue locally, so we will assume that we have the coordinate system from (3.3). We start with the first point. Choose lifts $g_{i} \in R_{T, n}$ of functions $f_{i} \in R_{n}$ for $1 \leqslant i \leqslant r$. We have

$$
\operatorname{res}_{T}\left(\left\{f_{1}, \ldots, f_{r}\right\}\right)=\operatorname{res}_{T}\left(\left(\operatorname{dlog} g_{1}, \log \left(g_{1}^{p} \varphi\left(g_{1}\right)^{-1}\right)\right) \cup \cdots \cup\left(\operatorname{dlog} g_{r}, \log \left(g_{r}^{p} \varphi\left(g_{r}\right)^{-1}\right)\right)\right)=0 .
$$

This proves the first equality of the first point of the lemma.

For the second equality of the same point, first assume $r=2$. We have (with an analogous notation)

$$
\begin{aligned}
\operatorname{res}_{T}(\{f, \varpi\}) & =\operatorname{res}_{T}\left(\left(\operatorname{dlog} g, \log \left(g^{p} \varphi(g)^{-1}\right)\right) \cup(\operatorname{dlog} T, 0)\right) \\
& =\operatorname{res}_{T}\left(\operatorname{dlog} g \operatorname{dlog} T, p \log \left(g^{p} \varphi(g)^{-1}\right) \operatorname{dlog} T\right) \\
& =\left(\operatorname{dlog} \bar{g}, p \log \left(\bar{g}^{p} \varphi(\bar{g})^{-1}\right)\right),
\end{aligned}
$$

where $\bar{g}$ is the reduction of $g$ to $R_{T, 0, n}$. Let $\alpha: \Omega_{D_{n}}^{\bullet} \rightarrow W_{n} \Omega_{R_{0}}^{\bullet}$ be the canonical map. Since the reduction of $\bar{g}$ to $R_{0}$ is the same as the reduction $\bar{f}$ of $f$, we can write $\alpha(\bar{g})=[\bar{f}] u, u \in 1+p W_{n} R_{0}$. We have $\operatorname{dlog} \alpha(\bar{g})=\operatorname{d} \log [\bar{f}]+\operatorname{dlog} u$. Set $c:=\bar{g}^{p} \varphi(\bar{g})^{-1}$. It follows that

$$
\begin{aligned}
\beta_{2}((\operatorname{dlog} \bar{g}, c)) & =(\operatorname{dlog}[\bar{f}]+\operatorname{dlog} u, \alpha(c))=(\operatorname{dlog}[\bar{f}], 0)+(\operatorname{dlog} u, \alpha(c)), \\
{\left[\beta_{2}((\operatorname{dlog} \bar{g}, c))\right] } & =\left[\beta_{1}(\operatorname{dlog}[\bar{f}])\right] .
\end{aligned}
$$

The last equality of cohomology classes can be seen using the computations in the first part of the proof of Lemma 3.7, and it finishes our argument. 


\section{Syntomic COHOMOLOGY AND $p$-ADiC MOTIVIC COHOMOLOGY}

For a general $r$, we compute similarly

$$
\begin{aligned}
\operatorname{res}_{T}\left(\left\{f_{1}, \ldots, f_{r-1}, \varpi\right\}\right) & =i^{*}\left(\left\{g_{1}, \ldots, g_{r-1}\right\}\right) \cup \operatorname{res}_{T}(\{\varpi\})=\left(\operatorname{dlog} \bar{g}_{1} \cdots \operatorname{dlog} \bar{g}_{r-1}, c\right) \cup(1,0) \\
& =\left(\operatorname{dlog} \bar{g}_{1} \cdots \operatorname{dlog} \bar{g}_{r-1}, c^{\prime}\right) .
\end{aligned}
$$

Here $i$ : Spec $R_{0} \hookrightarrow$ Spec $R_{T}$ is the natural map, and the first equality follows from the projection formula in crystalline cohomology. We define $c$ and $c^{\prime}$ by the second and the third equality, respectively. We get

$$
\begin{aligned}
\beta_{2}\left(\left(\operatorname{dlog} \bar{g}_{1} \cdots \operatorname{dlog} \bar{g}_{r-1}, c^{\prime}\right)\right) & =\left(\left(\left(\operatorname{dlog}\left[\bar{f}_{1}\right]+\operatorname{d} \log u_{1}\right) \cdots\left(\operatorname{dlog}\left[\bar{f}_{r-1}\right]+\operatorname{dlog} u_{r-1}\right), \alpha\left(c^{\prime}\right)\right)\right), \\
{\left[\beta_{2}\left(\left(\operatorname{dlog} \bar{g}_{1} \cdots \operatorname{dlog} \bar{g}_{r-1}, c^{\prime}\right)\right)\right] } & =\left[\beta_{1}\left(\operatorname{dlog}\left[\bar{f}_{1}\right] \cdots \operatorname{dlog}\left[\bar{f}_{r-1}\right]\right)\right],
\end{aligned}
$$

as wanted.

For the second point of the lemma, we start with sections $f_{i} \in M_{R}^{\mathrm{gp}}$ and $f_{i}=f_{i}^{\prime} X^{N_{i}}$, where $f_{i}^{\prime} \in R^{*}$ and, for $N_{i}=\left(m_{i, a+1}, \ldots, m_{i, d}\right) \in \mathbf{Z}^{d-a}$, we have $X^{N_{i}}:=\prod_{a+1 \leqslant j \leqslant d} X_{j}^{m_{i, j}}$. We get

$$
\operatorname{res}_{T}\left(\left\{f_{1}, \ldots, f_{r}\right\}\right)=\operatorname{res}_{T}\left(\left(\operatorname{dlog} g_{1}, c_{1}\right) \cup \cdots \cup\left(\operatorname{dlog} g_{r}, c_{r}\right)\right)=0 .
$$

Computing as above, we get

$$
\operatorname{res}_{T}\left(\left\{f_{1}, \ldots, f_{r-1}, \varpi\right\}\right)=\left(\operatorname{dlog} \bar{g}_{1} \cdots \operatorname{dlog} \bar{g}_{r-1}, c\right) .
$$

But $\operatorname{dlog} \alpha\left(\bar{g}_{i}\right)=\operatorname{d} \log \left(\left[\bar{f}_{i}^{\prime}\right] X^{N_{i}}\right)+\operatorname{dlog} u_{i}$ for $u_{i} \in 1+p W_{n} R_{0}$. Hence

$$
\begin{aligned}
{\left[\beta_{2}\left(\left(\operatorname{dlog} \bar{g}_{1} \cdots \operatorname{dlog} \bar{g}_{r-1}, c\right)\right)\right] } & =\left[\beta_{1}\left(\operatorname{dlog}\left(\left[\bar{f}_{1}^{\prime}\right] X^{N_{1}}\right) \cdots\left(\operatorname{dlog}\left(\left[\bar{f}_{r-1}^{\prime}\right] X^{N_{r-1}}\right)\right)\right)\right] \\
& =\left[\beta_{1}\left(\operatorname{dlog}\left[\bar{f}_{1}\right] \cdots \operatorname{dlog}\left[\bar{f}_{r-1}\right]\right)\right],
\end{aligned}
$$

as wanted.

Step 6: Globalization; the second triangle. To get the second triangle in the theorem, take the first triangle and push it down to the Nisnevich site. We obtain the $p^{N r}$-distinguished triangle

$$
\mathrm{R} \varepsilon_{*} \mathcal{S}_{n}^{\prime}(r)_{X} \rightarrow \mathrm{R} \varepsilon_{*} \mathcal{S}_{n}^{\prime}(r)_{X \times} \stackrel{\kappa_{N}}{\longrightarrow} \mathrm{R} \varepsilon_{*} W_{n} \Omega_{X_{0}, \log }^{r-1}[-r]
$$

Recall that, in the absence of a horizontal log-structure, Kato proved that $\tau_{\leqslant 0} \mathrm{R} \varepsilon_{*} W_{n} \Omega_{X_{0}, \log }^{r-1} \simeq$ $W_{n} \Omega_{X_{0}, \log }^{r-1}$ [Kat82]. This is also true in our setting: adding one horizontal irreducible divisor at a time, use Gysin sequences and Kato's original result. The second triangle in the theorem is just the truncation $\tau_{\leqslant r}$ of the above triangle, assuming, of course, that it is a $p^{N r}$-distinguished triangle, for $N$ as in the theorem. For that it suffices to check that the map $\mathcal{H}^{r}\left(\mathcal{S}_{n}^{\prime}(r)_{X^{\times}, \text {Nis }}\right) \rightarrow$ $W_{n} \Omega_{X_{0}, \log }^{r-1}$ is $p^{N r}$-surjective. But this follows from Lemma 3.8.

Remark 3.9. There is a variant of Theorem 3.5 in which $p^{N r}$ is replaced by a worse error $p^{N}$ for $N=N(p, e, r)$, but which has a slightly simpler proof. Namely, we can use the following commutative diagram in which rows are distinguished triangles:

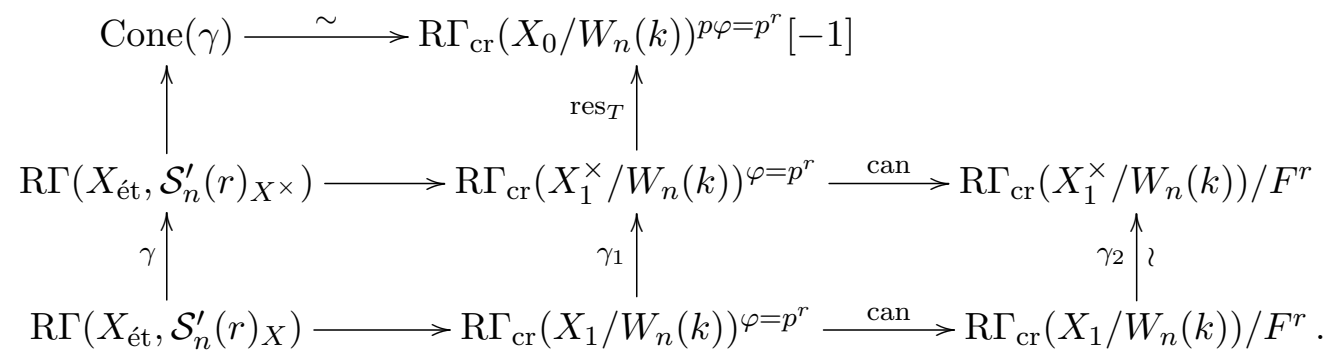




\section{ERTL AND W. NiziOŁ}

The first two columns are clearly distinguished triangles. The map $\gamma_{2}$ is a $p^{N}$-quasi-isomorphism for $N=N(p, e)$. In fact, we have canonical $p^{N}$-quasi-isomorphisms

$$
\begin{aligned}
\mathrm{R} \Gamma_{\mathrm{cr}}\left(X_{1}^{\times} / W_{n}(k)\right) / F^{r} & \rightarrow \mathrm{R} \Gamma_{\mathrm{cr}}\left(X_{1}^{\times} / \mathcal{O}_{K, n}^{\times}\right) / F^{r}, \\
\mathrm{R} \Gamma_{\mathrm{cr}}\left(X_{1} / W_{n}(k)\right) / F^{r} & \rightarrow \mathrm{R} \Gamma_{\mathrm{cr}}\left(X_{1} / \mathcal{O}_{K, n}\right) / F^{r} .
\end{aligned}
$$

The first one is proved in the proof of [NN16, Corollary 2.4]; the second one is proved by a nonlogarithmic version of the same argument. It follows that the top horizontal map in the above diagram is a $p^{N}$-quasi-isomorphism for $N=N(p, e)$. It now suffices to construct a $p^{N}$-quasiisomorphism, for $N=N(r)$, between the complexes of sheaves $W_{n} \Omega_{X_{0}, \log }^{r-1}[-r+1]$ and $\mathcal{A}_{\mathrm{cr}, n}^{p \varphi=p^{r}}$; this was done in the proof of Theorem 3.5.

Corollary 3.10. Let $X$ be a semistable scheme over $\mathcal{O}_{K}$ with a smooth special fiber. There exist a constant $N=N(p, e, d, r)$ and the following natural family of compatible $p^{m}$-quasiisomorphisms for $m \geqslant N$, where * denotes the étale or Nisnevich topology of $X$,

$$
\gamma(m): \mathcal{S}_{n}^{\prime}(r)_{X, *} \oplus W_{n} \Omega_{X_{0}, \log }^{r-1}[-r] \rightarrow \mathcal{S}_{n}^{\prime}(r)_{X^{\times}, *} \cdot
$$

Proof. It suffices to argue in the étale topology. The commutative diagram below shows that there

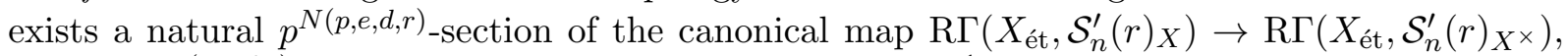
hence a $p^{N(p, e, d, r)}$-section of the map $\mathcal{S}_{n}^{\prime}(r)_{X^{\times} \text {,ét }} \rightarrow W_{n} \Omega_{X_{0}, \log }^{r-1}[-r]$, as wanted:

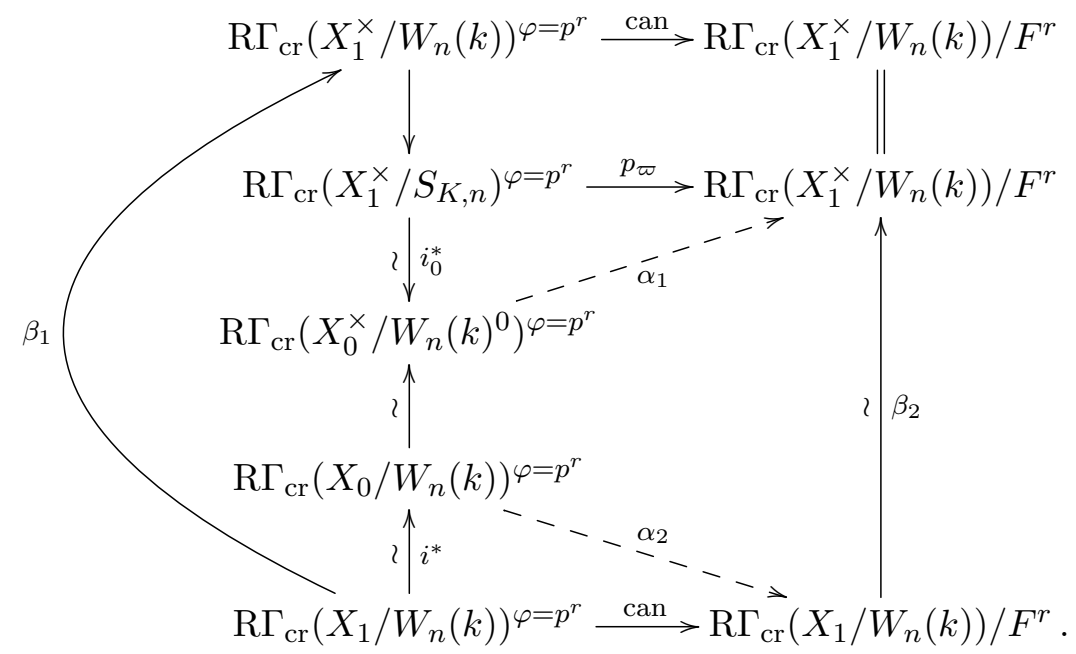

Here the map $p_{\varpi}$ is induced by $T \mapsto \varpi$, the map $i_{0}^{*}$ by $T \mapsto 0$. The latter map is a quasiisomorphism: this is an immediate consequence of the fact that the Frobenius morphism is highly topologically nilpotent on the divided power ideal of $S_{K}$. The map $\alpha_{1}$ is defined to make the triangle commute. The map $\alpha_{2}$ is defined to make the trapezoid $p^{N(p, e, d)}$-commute: recall that $\beta_{2}$ is a $p^{N(p, e)}$-quasi-isomorphism, and use Section 3.1.2. The map $i^{*}$, where $i: X_{0} \hookrightarrow X_{1}$ is the natural closed immersion, is a $p^{N(p, e)}$-quasi-isomorphism [CN17, proof of Lemma 5.9]. A diagram chase shows that the bottom triangle $p^{N(p, e, d)}$-commutes.

Let, for $*$ denoting the étale or the Nisnevich topology,

$$
\begin{aligned}
\mathrm{R} \Gamma\left(X_{*}, \mathcal{S}(r)\right)_{\mathbf{Q}} & :=\operatorname{holim}_{n} \mathrm{R} \Gamma\left(X_{*}, \mathcal{S}_{n}(r)\right) \otimes \mathbf{Q} \stackrel{\sim}{\rightarrow} \operatorname{holim}_{n} \mathrm{R} \Gamma\left(X_{*}, \mathcal{S}_{n}^{\prime}(r)\right) \otimes \mathbf{Q}, \\
\mathrm{R} \Gamma\left(X_{*}, \mathcal{E}(r)\right)_{\mathbf{Q}} & :=\operatorname{holim}_{n} \mathrm{R} \Gamma\left(X_{*}, \mathcal{E}_{n}(r)\right) \otimes \mathbf{Q} \stackrel{\sim}{\rightarrow} \operatorname{holim}_{n} \mathrm{R} \Gamma\left(X_{*}, \mathcal{E}_{n}^{\prime}(r)\right) \otimes \mathbf{Q}, \\
\mathrm{R} \Gamma\left(X_{*}, W \Omega_{X_{0}, \log }^{r-1}\right)_{\mathbf{Q}} & :=\operatorname{holim}_{n} \mathrm{R} \Gamma\left(X_{*}, i_{*} W_{n} \Omega_{X_{0}, \log }^{r-1}\right) .
\end{aligned}
$$




\section{SyNTOMIC COHOMOLOGY AND $p$-ADIC MOTIVIC COHOMOLOGY}

In the proof of Theorem 3.5, we have shown that there is a $p^{N(d) r}$-quasi-isomorphism

$$
W_{n} \Omega_{X_{0}, \log }^{r-1}[-r+1] \stackrel{\sim}{\rightarrow}\left[\mathcal{A}_{\mathrm{cr}, n} \stackrel{p^{r}-p \varphi}{\longrightarrow} \mathcal{A}_{\mathrm{cr}, n}\right] .
$$

It follows that we have

$$
\mathrm{R} \Gamma\left(X_{*}, W \Omega_{X_{0}, \log }^{r-1}\right)_{\mathbf{Q}} \simeq \mathrm{R} \Gamma_{\mathrm{cr}}\left(X_{0} / F\right)_{\mathbf{Q}}^{\varphi=p^{r-1}}[r-1],
$$

where, for a scheme $Y$ over $W(k)$, we set

$$
\mathrm{R} \Gamma_{\mathrm{cr}}(Y / F):=\mathrm{R} \Gamma_{\mathrm{cr}}(Y / W(k))_{\mathbf{Q}}:=\operatorname{holim}_{n} \mathrm{R} \Gamma_{\mathrm{cr}}\left(Y_{1} / W_{n}(k)\right) \otimes \mathbf{Q} .
$$

The following corollary is an immediate consequence of Corollary 3.10.

Corollary 3.11. Let $X$ be a semistable scheme over $\mathcal{O}_{K}$ with a smooth special fiber. We have the following natural quasi-isomorphisms:

$$
\mathrm{R} \Gamma\left(X_{*}, \mathcal{S}(r)\right)_{\mathbf{Q}} \oplus \mathrm{R} \Gamma\left(X_{*}, W \Omega_{X_{0}, \log }^{r-1}\right)_{\mathbf{Q}}[-r] \stackrel{\sim}{\rightarrow} \mathrm{R} \Gamma\left(X_{*}^{\times}, \mathcal{S}(r)\right)_{\mathbf{Q}} .
$$

Corollary 3.12. Let $X$ be a semistable scheme over $\mathcal{O}_{K}$ with a smooth special fiber. For a constant $N=N(p, d)$ or $N=N(p, d, e)$, depending on whether $K$ has enough roots of unity or not, we have the following family of compatible $p^{m r}$-distinguished triangles, for $m \geqslant N$, of sheaves in the étale topology of $X_{0}$ :

$$
\mathcal{S}_{n}^{\prime}(r)_{X} \rightarrow \tau_{\leqslant r} i^{*} \mathrm{R} j_{*} \mathbf{Z} / p^{n}(r)^{\prime} \rightarrow W_{n} \Omega_{X_{0}, \log }^{r-1}[-r] .
$$

Moreover, for a constant $N=N(p, e, d, r)$, we have the following $p^{m}$-quasi-isomorphisms for $m \geqslant N$ :

$$
\gamma(m): \mathcal{S}_{n}^{\prime}(r)_{X} \oplus W_{n} \Omega_{X_{0}, \log }^{r-1}[-r] \rightarrow \tau_{\leqslant r} i^{*} \mathrm{R} j_{*} \mathbf{Z} / p^{n}(r)^{\prime} .
$$

Proof. This immediately follows from Theorems 3.5 and 2.3 and Corollary 3.10.

Remark 3.13. For $r \leqslant p-2$, the distinguished triangle (3.16) was constructed before by Kurihara. No additional constants are needed in this case.

Theorem 3.14 ([Kur87, 1]). Let $X$ be a smooth scheme over $\mathcal{O}_{K}$. For $r \leqslant p-2$, we have the following distinguished triangle of sheaves in the étale topology of $X_{0}$ :

$$
\mathcal{S}_{n}(r)_{X} \rightarrow \tau_{\leqslant r} i^{*} \mathrm{R} j_{*} \mathbf{Z} / p^{n}(r) \rightarrow W_{n} \Omega_{X_{0}, \log }^{r-1}[-r] .
$$

It is easy to see that Theorem 3.14 holds also for schemes $X$ that are semistable over $\mathcal{O}_{K}$ with a smooth special fiber, that is, that we have the distinguished triangle

$$
\mathcal{S}_{n}(r)_{X} \rightarrow \tau_{\leqslant r} i^{*} \mathrm{R} j_{*} \mathbf{Z} / p^{n}(r) \rightarrow W_{n} \Omega_{X_{0}, \log }^{r-1}[-r], \quad r \leqslant p-2 .
$$

Indeed, it suffices to note that all the terms involved have Gysin sequences that are compatible with the maps in the sequence [Tsu00] and to use Theorem 3.14. In particular, in view of Theorem 2.2, we have the distinguished triangle

$$
\mathcal{S}_{n}(r)_{X} \rightarrow \mathcal{S}_{n}(r)_{X^{\times}} \rightarrow W_{n} \Omega_{X_{0}, \log }^{r-1}[-r], \quad r \leqslant p-2,
$$

a "small twists" analog of the distinguished triangles from Theorem 3.5.

\subsection{Syntomic-étale cohomology and motivic cohomology}

The main theorem of this section shows that, in étale topology, syntomic-étale complexes on smooth schemes over $\mathcal{O}_{K}$ approximate motivic complexes. 


\section{ERTL AND W. NiZIOE}

Theorem 3.15. Let $X$ be a semistable scheme over $\mathcal{O}_{K}$ with a smooth special fiber. We treat it as a log-scheme with the log-structure induced by the divisor at infinity. Let $j^{\prime}: X_{\operatorname{tr}} \hookrightarrow X$ be the natural open immersion.

(i) There is a natural cycle class map

$$
\mathrm{cl}_{r}^{\mathrm{syn}}: \mathrm{R} j_{*}^{\prime} \mathbf{Z} / p^{n}(r)_{\mathrm{M}} \rightarrow \mathcal{E}_{n}(r)_{X, \mathrm{Nis}}, \quad 0 \leqslant r \leqslant p-2 .
$$

It is a quasi-isomorphism.

(ii) There exist a constant $N=N(p, d)$ or $N=N(p, e, d)$, depending on whether $K$ has enough roots of unity or not, and a family of natural and compatible cycle class maps, for $m \geqslant N$,

$$
\mathrm{cl}_{r}^{\mathrm{syn}, m}: \mathrm{R} j_{*}^{\prime} \mathbf{Z} / p^{n}(r)_{\mathrm{M}} \rightarrow \mathcal{E}_{n}^{\prime}(r)_{X, \mathrm{Nis}}, \quad r \geqslant 0,
$$

that are $p^{m r}$-quasi-isomorphisms.

We have analogous statements in the étale topology. These cycle class maps are compatible (via the localization map and the period map) with the twisted étale cycle class maps; that is, we have the commutative diagram

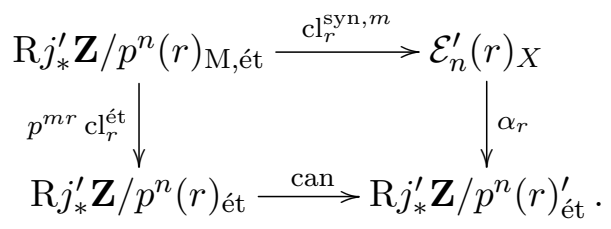

Proof. We will define the classes $\mathrm{cl}_{r}^{\mathrm{syn}, N}$, for a constant $N$ as in the theorem, and $\mathrm{set}_{\mathrm{cl}}^{\mathrm{syn}, m}=$ $p^{(m-N) r} \mathrm{cl}_{r}^{\mathrm{syn}, N}$ for $m \geqslant N$.

We start with the Nisnevich topology. We will prove the second claim, the proof of the first one being analogous. Consider the commutative diagram

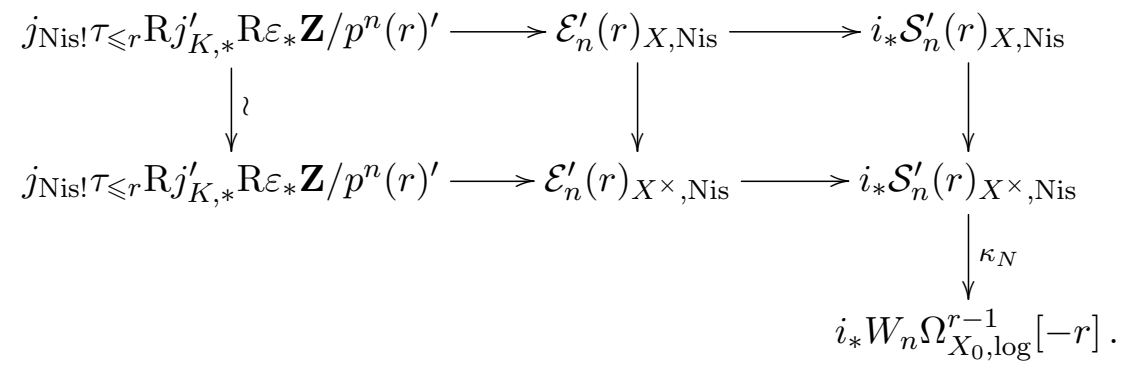

The two rows are distinguished triangles; the right column is a $p^{N r}$-distinguished triangle, for a constant $N$ as in the theorem, by Theorem 3.5. It follows that for the same type of $N$, we have the $p^{N r}$-distinguished triangle

$$
\mathcal{E}_{n}^{\prime}(r)_{X, \mathrm{Nis}} \rightarrow \mathcal{E}_{n}^{\prime}(r)_{X \times, \mathrm{Nis}} \stackrel{\kappa_{N}}{\longrightarrow} i_{*} W_{n} \Omega_{X_{0}, \log }^{r-1}[-r] .
$$

Let $Y=X_{\mathrm{tr}}$. By functoriality, we get the map of $p^{N r}$-distinguished triangles

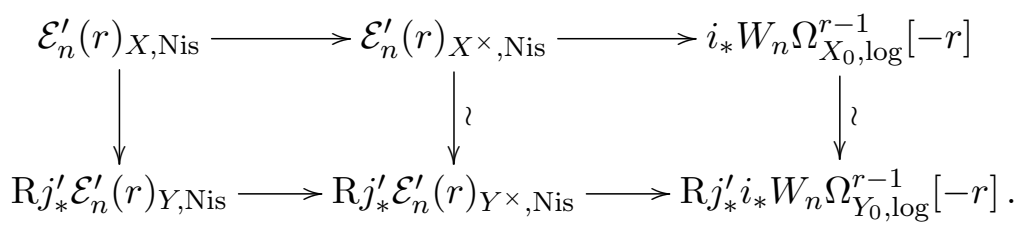




\section{SyNTOMIC COHOMOLOGY AND $p$-ADIC MOTIVIC COHOMOLOGY}

The right vertical arrow is a quasi-isomorphism since $M_{X_{0}}=j_{*}^{\prime} \mathcal{O}_{X_{0}, \mathrm{tr}}^{*}$ : the Gysin sequence for logarithmic de Rham-Witt cohomology implies the first isomorphism below [Sat13, 2.1.1],

$$
W_{n} \Omega_{X_{0}, \log }^{r-1} \stackrel{\sim}{\rightarrow} j_{*}^{\prime} W_{n} \Omega_{Y_{0}, \log }^{r-1} \stackrel{\sim}{\rightarrow} \mathrm{R} j_{*}^{\prime} W_{n} \Omega_{Y_{0}, \log }^{r-1} ;
$$

the second one follows from the quasi-isomorphisms $W_{n} \Omega_{Y_{0}, \log }^{r-1} \simeq j_{*}^{\prime} \mathbf{Z} / p^{n}(r-1)_{M}$ and $j_{*}^{\prime} \mathbf{Z} / p^{n}(r-$ $1)_{M} \stackrel{\sim}{\rightarrow} \mathrm{R} j_{*}^{\prime} \mathbf{Z} / p^{n}(r-1)_{M}$. The middle vertical arrow is a $p^{N r}$-quasi-isomorphism by Corollary 2.6. Hence, the left vertical arrow is a $p^{N r}$-quasi-isomorphism, and we may assume that the horizontal divisor of $X$ is trivial.

Consider the diagram

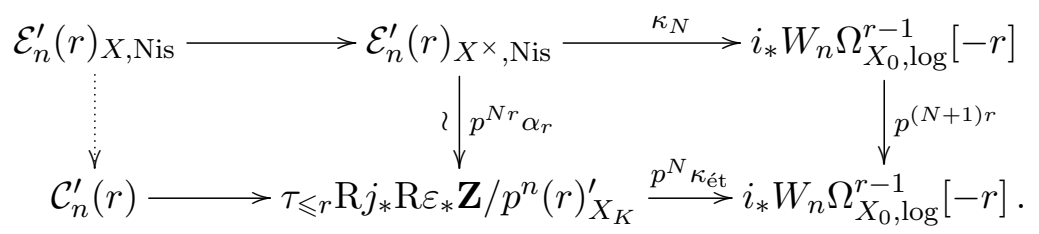

Here, the map $\kappa_{\text {ét }}$ is induced from a map $\tau_{\leqslant r} i^{*} \mathrm{R} j_{*} \mathbf{Z} / p^{n}(r) \rightarrow W_{n} \Omega_{X_{0}, \log }^{r-1}[-r]$ of sheaves on the étale site of $X_{0}$ defined as the composition of the canonical map

$$
\tau_{\leqslant r} i^{*} \mathrm{R} j_{*} \mathbf{Z} / p^{n}(r) \rightarrow i^{*} \mathrm{R}^{r} j_{*} \mathbf{Z} / p^{n}(r)[-r]
$$

and the symbol map $i^{*} \mathrm{R}^{r} j_{*} \mathbf{Z} / p^{n}(r) \rightarrow W_{n} \Omega_{X_{0}, \log }^{r-1}$. The latter is defined by observing that $i^{*} \mathrm{R}^{r} j_{*} \mathbf{Z} / p^{n}(r)$ is locally generated by symbols $\left\{f_{1}, \ldots, f_{r}\right\}$ for $f_{i} \in i^{*} j_{*} \mathcal{O}_{X_{K}}^{*}$ [BK86, Corollary 6.1.1]. By multilinearity, each symbol can be written as a sum of symbols of the form $\left\{f_{1}, \ldots, f_{r}\right\}$ and $\left\{f_{1}, \ldots, f_{r-1}, \varpi\right\}$ for $f_{i} \in i^{*} \mathcal{O}_{X}^{*}$. Then $\kappa_{\text {ét }}$ sends the former to zero and the latter to $\operatorname{dlog}\left[\bar{f}_{1}\right] \cdots \operatorname{dlog}\left[\bar{f}_{r-1}\right]$, where $\bar{f}_{i}$ is the reduction of $f_{i}$ to $\mathcal{O}_{X_{0}}^{*}$. We define $\mathcal{C}_{n}^{\prime}(r)$ as the mapping fiber of the map $p^{N} \kappa_{\text {ét }}$.

We claim that the right square of the diagram commutes. Indeed, we note that we can pass to the étale site, and there it suffices to show that the following diagram of maps of sheaves commutes:

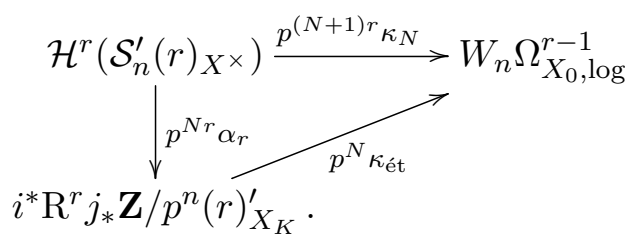

Since the map $\alpha_{r}$ is a $p^{N r}$-isomorphism, it is compatible with symbols up to $p^{r}$-twits; that is, $\alpha_{r}$ maps a symbol to the same symbol times $p^{r}$, and the sheaf $i^{*} \mathrm{R}^{r} j_{*} \mathbf{Z} / p^{n}(r)_{X_{K}}$ is generated locally by symbols. It suffices to check that the map $\kappa_{N}$ sends the symbol $\left\{f_{1}, \ldots, f_{r}\right\}$, for $f_{i} \in i^{*} \mathcal{O}_{X}^{*}$, to 0 and the symbol $\left\{f_{1}, \ldots, f_{r-1}, \varpi\right\}$, for $f_{i} \in i^{*} \mathcal{O}_{X}^{*}$, to $p^{N} \operatorname{dlog}\left[\bar{f}_{1}\right] \cdots \operatorname{dlog}\left[\bar{f}_{r-1}\right]$. But this follows from Lemma 3.8. It follows that the left vertical map in the diagram (3.18) exists. It is unique because

$$
\operatorname{Hom}\left(\mathcal{E}_{n}^{\prime}(r)_{X, \mathrm{Nis}}, W_{n} \Omega_{X_{0}, \log }^{r-1}[-r-1]\right)=0
$$

for degree reasons. It is clearly a quasi-isomorphism.

It now remains to show that there exists a $p^{N r}$-quasi-isomorphism $\mathbf{Z} / p^{n}(r)_{M} \rightarrow \mathcal{C}_{n}^{\prime}(r)$ for $N$ as in the theorem. We proceed as in [Gei04, Section 6]. Consider the following diagram of distinguished triangles (the complex $\mathcal{C}_{n}(r)$ is defined by the bottom triangle and is $p^{N r}$-quasi- 
isomorphic to the complex $\left.\mathcal{C}_{n}^{\prime}(r)\right)$ :

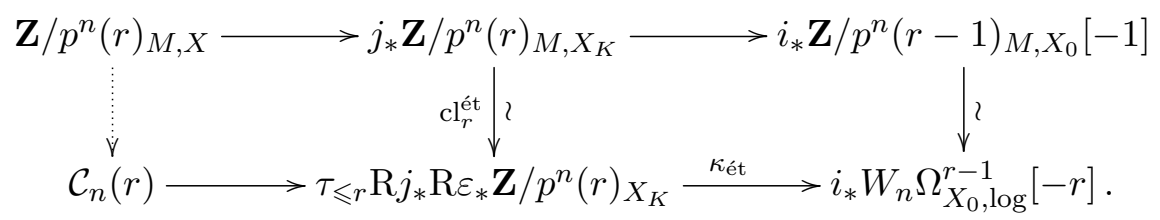

The middle and the right vertical maps are induced by the étale and logarithmic de Rham-Witt cycle class map, respectively. They are quasi-isomorphisms by the Beilinson-Lichtenbaum conjecture. The right square commutes: pass to the étale site; there, this fact was shown in [Gei04, Section 6]. Hence, the left vertical map exists, is unique, and is a quasi-isomorphism as well. This concludes the proof of our theorem.

For the étale topology, the computations are analogous, but the diagram (3.19) has to be replaced with the following one:

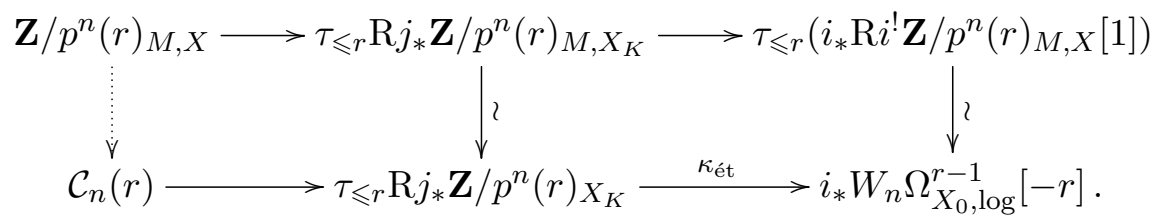

The right vertical arrow is a quasi-isomorphism by [Gei04, Section 6].

Consider the composition of maps defined above,

$$
\mathrm{R} j_{*}^{\prime} \mathbf{Z} / p^{n}(r)_{M, Y} \rightarrow \mathrm{R} j_{*}^{\prime} \mathcal{C}_{n}^{\prime}(r) \leftarrow \mathrm{R} j_{*}^{\prime} \mathcal{E}_{n}^{\prime}(r)_{Y, \mathrm{Nis}} \leftarrow \mathcal{E}_{n}^{\prime}(r)_{X, \mathrm{Nis}},
$$

where we invert the last two maps (in the $p^{N r}$-sense). By the above, it is a $p^{N r}$-quasi-isomorphism for $N$ as in the theorem. We choose one such $N$ and set $\mathrm{cl}_{r}^{\mathrm{syn}, N}$ equal to that composition. The claimed compatibility with the étale cycle class follows easily from the definitions.

We list several, more or less immediate, corollaries of Theorems 3.14 and 3.15 (we set $\alpha:=$ ét, Nis).

Corollary 3.16. Let $X$ be a smooth scheme over $\mathcal{O}_{K}$.

(i) We have $H_{\alpha}^{*}\left(X, \mathcal{E}_{n}(r)\right) \simeq H_{\mathrm{M}, \alpha}^{*}\left(X, \mathbf{Z} / p^{n}(r)\right)$ for $r \leqslant p-2$.

(ii) The kernel and cokernel of the cycle class map

$$
\operatorname{cl}_{n}^{\mathrm{syn}, N}: H_{\mathrm{M}, \alpha}^{*}\left(X, \mathbf{Z} / p^{n}(r)\right) \rightarrow H_{\alpha}^{*}\left(X, \mathcal{E}_{n}^{\prime}(r)\right)
$$

are annihilated by $p^{N r}$, where $N$ denotes the constant from Theorem 3.15. Hence,

$$
H_{\alpha}^{*}(X, \mathcal{E}(r))_{\mathbf{Q}} \simeq H_{\mathrm{M}, \alpha}^{*}\left(X, \mathbf{Q}_{p}(r)\right) .
$$

In the more familiar language of syntomic cohomology, Theorem 3.15 and Corollary 3.16 can be stated in the following way.

Corollary 3.17. Let $X$ be a semistable scheme over $\mathcal{O}_{K}$ with a smooth special fiber. Let $j^{\prime}: X_{\operatorname{tr}} \hookrightarrow X$ be the natural open immersion. Then, on the étale site of $X_{0}$,

(i) there is a natural quasi-isomorphism [Gei04]

$$
\mathcal{S}_{n}(r)_{X} \simeq i^{*} \mathrm{R} j_{*}^{\prime} \mathbf{Z} / p^{n}(r)_{\mathrm{M}}, \quad 0 \leqslant r \leqslant p-2 ;
$$


(ii) there exist a constant $N$ as in Theorem 3.15 and a natural $p^{N r}$-quasi-isomorphism

$$
\mathcal{S}_{n}^{\prime}(r)_{X} \simeq i^{*} \mathrm{R} j_{*}^{\prime} \mathbf{Z} / p^{n}(r)_{\mathrm{M}}^{\prime}, \quad r \geqslant 0 .
$$

Corollary 3.18. Let $X$ be a proper semistable scheme over $\mathcal{O}_{K}$ with a smooth special fiber.

(i) We have $H_{\alpha}^{*}\left(X, \mathcal{S}_{n}(r)\right) \simeq H_{\mathrm{M}, \alpha}^{*}\left(X_{\mathrm{tr}}, \mathbf{Z} / p^{n}(r)\right)$ for $r \leqslant p-2$.

(ii) The kernel and cokernel of the cycle class map

$$
H_{\mathrm{M}, \alpha}^{*}\left(X_{\mathrm{tr}}, \mathbf{Z} / p^{n}(r)\right) \rightarrow H_{\alpha}^{*}\left(X, \mathcal{S}_{n}^{\prime}(r)\right)
$$

are annihilated by $p^{N r}$, where $N$ denotes the constant from Theorem 3.15. Hence,

$$
H_{\alpha}^{*}(X, \mathcal{S}(r))_{\mathbf{Q}} \simeq H_{\mathrm{M}, \alpha}^{*}\left(X_{\mathrm{tr}}, \mathbf{Q}_{p}(r)\right) .
$$

Corollary 3.19. Let $X$ be a proper semistable scheme over $\mathcal{O}_{K}$ with a smooth special fiber. Then the claims of Corollary 3.18 hold for $X_{\mathcal{O}_{\bar{K}}}$ (in place of $X$ ). Moreover, for $i \leqslant r$, we have the commutative diagram

$$
\begin{aligned}
& H_{\mathrm{M}}^{i}\left(X_{\mathcal{O}_{\bar{K}}, \mathrm{tr}}, \mathbf{Q}_{p}(r)\right) \stackrel{j^{*}}{\sim} H_{\mathrm{M}}^{i}\left(X_{\bar{K}, \mathrm{tr}}, \mathbf{Q}_{p}(r)\right) \\
& \text { ? } \mathrm{cl}_{i, r}^{\mathrm{syn}} \quad \text { ? } p^{(N-1) r} \mathrm{cl}_{i, r}^{\mathrm{e} t} \\
& H_{\text {ét }}^{i}\left(X_{\mathcal{O}_{\bar{K}}}, \mathcal{S}^{\prime}(r)\right)_{\mathbf{Q}} \stackrel{p^{-r} \alpha_{i, r}}{\longrightarrow} H_{\text {ét }}^{i}\left(X_{\bar{K}, \mathrm{tr}}, \mathbf{Q}_{p}(r)\right) \text {. }
\end{aligned}
$$

Note. The syntomic cohomology of $X_{\mathcal{O}_{\bar{K}}}$ is defined in the same way as that of $X$; see [Bei13, 1.18] for details.

Proof. The first and the second claims follow from Corollary 3.18 and Theorem 3.15 by passing to the limit over finite extensions of $K$ in $\bar{K}$. The fact that the localization map $j^{*}$ is an isomorphism was proved in [Niz98, Lemma 3.1].

Remark 3.20. For $X$ proper, the above diagram was studied in [Niz98] (see [Niz06] for a brief survey): it was constructed first for the Chern classes from $p$-adic $K$-theory and then for motivic cohomology by studying the compatibility of Chern classes with operations on $K$-theory. This did not use the Fontaine-Messing period map $\alpha_{i, r}$; instead, a period map $\alpha_{i, r}: H_{\text {ét }}^{i}\left(X_{\bar{K}, \mathrm{tr}}, \mathbf{Q}_{p}(r)\right) \rightarrow$ $H_{\text {ét }}^{i}\left(X_{\mathcal{O}_{\bar{K}}}, \mathcal{S}(r)\right)_{\mathbf{Q}}$ was defined using the above diagram. The fact that it is an isomorphism followed from the proof of the crystalline conjecture and implies that the syntomic cycle class map $\mathrm{cl}_{i, r}^{\mathrm{syn}}$ is also an isomorphism.

For an open $X$ as above, the situation is, at the moment, reversed. We defined log-syntomic $p$-adic Chern classes [Niz16] using the (universal) syntomic cycle class maps constructed in this paper.

\section{Appendix. Comparison of crystalline, convergent, and rigid syntomic cohomologies}

We will compare the crystalline, the convergent, and the rigid syntomic cohomology for smooth schemes over $\mathcal{O}_{K}$ with normal crossing compactifications. Let $X$ be a smooth scheme over $\mathcal{O}_{K}$. Recall Besser's definition of rigid syntomic cohomology [Bes00]:

$$
\mathrm{R} \Gamma_{\text {syn }}^{\text {rig }}(X, r):=\left[\mathrm{R} \Gamma_{\text {rig }}\left(X_{0} / F\right) \oplus F^{r} \mathrm{R} \Gamma_{\mathrm{dR}}\left(X_{K}\right) \stackrel{f}{\longrightarrow} \mathrm{R} \Gamma_{\text {rig }}\left(X_{0} / F\right) \oplus \mathrm{R} \Gamma_{\text {rig }}\left(X_{0} / K\right)\right], \quad r \geqslant 0 .
$$

Here $\mathrm{R} \Gamma_{\text {rig }}(\cdot)$ denotes the rigid cohomology complex, and $f$ is the map defined by $(x, y) \mapsto$ $\left(\left(p^{r}-\varphi\right)(x), \operatorname{sp}(y)-x\right)$, where sp is Berthelot's specialization map. 


\section{ERTL AND W. NiziOŁ}

Proposition A.1. Let $X$ be a proper semistable scheme over $\mathcal{O}_{K}$ with a smooth special fiber. There is a natural quasi-isomorphism

$$
\mathrm{R} \Gamma_{\mathrm{syn}}^{\mathrm{rig}}\left(X_{\mathrm{tr}}, r\right) \simeq \mathrm{R} \Gamma_{\mathrm{syn}}(X, r), \quad r \geqslant 0 .
$$

Proof. As usual, we consider $X$ as a log-scheme (with a trivial vertical log-structure). We can write

$$
\mathrm{R} \Gamma_{\mathrm{syn}}^{\mathrm{rig}}\left(X_{\mathrm{tr}}, r\right) \simeq\left[\mathrm{R} \Gamma_{\text {rig }}\left(X_{0, \mathrm{tr}} / F\right)^{\varphi=p^{r}} \rightarrow \mathrm{R} \Gamma_{\text {rig }}\left(X_{0, \mathrm{tr}} / K\right) / F^{r} \mathrm{R} \Gamma_{\mathrm{dR}}\left(X_{K, \mathrm{tr}}\right)\right] .
$$

Since we have

$$
\mathrm{R} \Gamma_{\mathrm{syn}}(X, r) \simeq\left[\mathrm{R} \Gamma_{\mathrm{cr}}(X / F)^{\varphi=p^{r}} \rightarrow \mathrm{R} \Gamma_{\mathrm{dR}}\left(X_{K}\right) / F^{r}\right]
$$

it suffices to construct a map

$$
\mathrm{R} \Gamma_{\mathrm{cr}}(X / F) \rightarrow \mathrm{R} \Gamma_{\text {rig }}\left(X_{0, \operatorname{tr}} / F\right)
$$

that is compatible (in the dg-category sense) with the Frobenius morphism and the specialization map from de Rham cohomology. This is accomplished by the following commutative diagram:

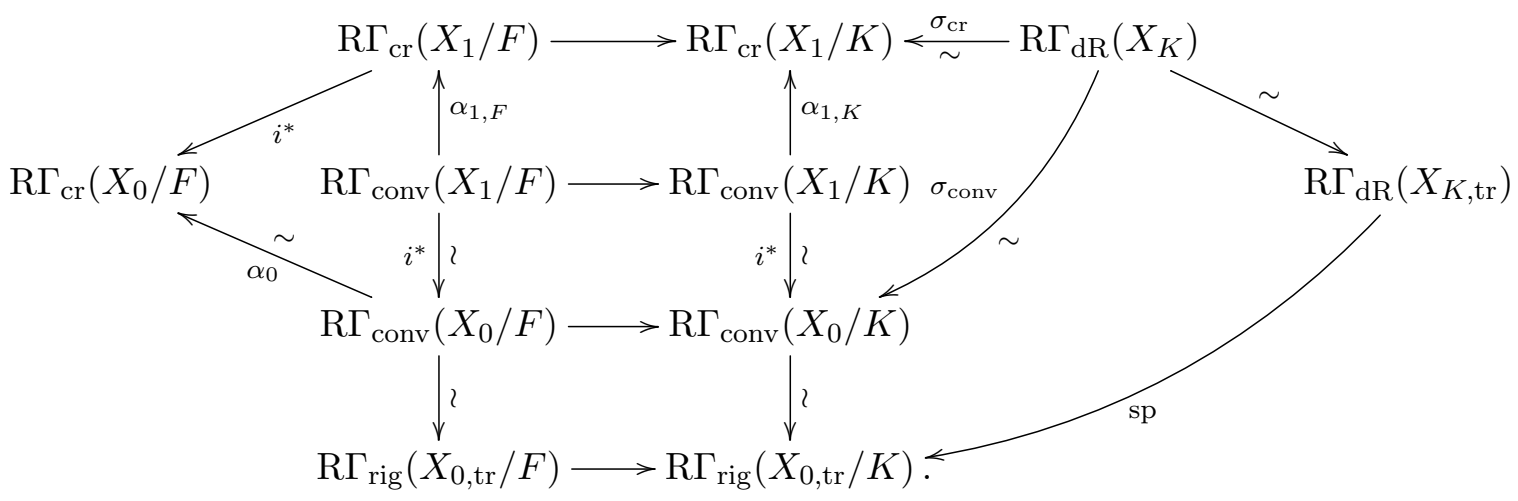

Here $\mathrm{R} \Gamma_{\text {conv }}(\cdot)$ denotes the (logarithmic) convergent cohomology [Ogu90, BO83, Shi02] that is used classically to connect rigid cohomology with crystalline cohomology. The quasi-isomorphisms between the rigid and the convergent cohomology at the bottom of the diagram are proved in [Shi02, Corollary 2.4.13]. The maps $i^{*}$ are quasi-isomorphisms by the invariance of convergent cohomology under nilpotent thickenings [BO83, 1.14.3]. The map $\alpha_{0}$ is a quasi-isomorphism by [Shi02, Theorem 3.1.1]. The top map $i^{*}$ is a quasi-isomorphism on $\varphi$-eigenspaces [CN17, proof of Lemma 5.9]; hence, so is the map $\alpha_{1, F}$. The maps $\sigma_{\text {cr }}$ and $\sigma_{\text {conv }}$ are quasi-isomorphisms simply by the crystalline and the convergent [Shi02, 2.3] Poincaré lemmas, respectively. It follows that the specialization map sp and the map $\alpha_{1, K}$ are quasi-isomorphisms as well.

Remark A.2. Recall that Besser's definition of rigid syntomic cohomology is modeled on the definition of convergent syntomic cohomology [Niz01]. In its logarithmic form, the latter is defined as the mapping fiber

$$
\mathrm{R} \Gamma_{\text {syn }}^{\text {conv }}(X, r):=\left[\mathrm{R} \Gamma_{\text {conv }}\left(X_{0} / F\right)^{\varphi=p^{r}} \rightarrow \mathrm{R} \Gamma_{\text {conv }}\left(X_{0} / K\right) / F^{r} \mathrm{R} \Gamma_{\text {conv }}\left(X_{0} / K\right)\right] .
$$

The proof of Proposition A.1 shows that for a proper and semistable scheme over $\mathcal{O}_{K}$ with a smooth special fiber, we have natural quasi-isomorphisms

$$
\mathrm{R} \Gamma_{\mathrm{syn}}^{\mathrm{rig}}\left(X_{\mathrm{tr}}, r\right) \simeq \mathrm{R} \Gamma_{\mathrm{syn}}^{\mathrm{conv}}(X, r) \simeq \mathrm{R} \Gamma_{\mathrm{syn}}(X, r), \quad r \geqslant 0 .
$$

In the proper case, this was shown in [Bes00, Proposition 9.8].

For a variety $Y$ over $K$, let $\mathrm{R} \Gamma_{\mathrm{syn}}^{N N}(Y, r)$ denote the syntomic cohomology defined in [NN16]. 


\section{SyNTOMIC COHOMOLOGY AND $p$-ADIC MOTIVIC COHOMOLOGY}

Corollary A.3. Let $X$ be a proper semistable scheme over $\mathcal{O}_{K}$ with a smooth special fiber. There is a natural distinguished triangle

$$
\mathrm{R} \Gamma_{\mathrm{syn}}^{\mathrm{rig}}\left(X_{\mathrm{tr}}, r\right) \oplus \mathrm{R} \Gamma\left(X_{0, \text { ét }}, W \Omega_{X_{0}, \log }^{r-1}\right)_{\mathbf{Q}}[-r] \stackrel{\sim}{\rightarrow} \mathrm{R} \Gamma_{\mathrm{syn}}^{N N}\left(X_{K, \mathrm{tr}}, r\right) .
$$

Proof. Since we have a canonical quasi-isomorphism [NN16, Proposition 3.18]

$$
\mathrm{R} \Gamma_{\mathrm{syn}}\left(X^{\times}, r\right)_{\mathbf{Q}} \stackrel{\sim}{\rightarrow} \mathrm{R} \Gamma_{\mathrm{syn}}^{N N}\left(X_{K, \mathrm{tr}}, r\right),
$$

this follows immediately from Proposition A.1 and Corollary 3.11.

\section{ACKNOWLEDGEMEnTS}

We thank the referee for a very careful reading of the manuscript and helpful comments. We would like to thank Grzegorz Banaszak, Pierre Colmez, Christian Haesemeyer, and Bruno Kahn for discussions related to the content of this paper.

\section{REFERENCES}

Bei13 A. Beilinson, On the crystalline period map, 2013, arXiv:1111.3316. This is an extended version of Camb. J. Math. 1 (2013), no. 1, 1-51; doi:10.4310/CJM.2013.v1.n1.a1.

Bes00 A. Besser, Syntomic regulators and p-adic integration. I. Rigid syntomic regulators, Israel J. Math. 120 (2000), part B, 291-334; doi:10.1007/BF02834843.

BK86 S. Bloch and K. Kato, p-adic étale cohomology, Publ. Math. Inst. Hautes Études Sci. (1986), no. 63, 107-152; doi:10.1007/BF02831624.

Blo86 S. Bloch, Algebraic cycles and higher K-theory, Adv. Math. 61 (1986), no. 3, 267-304; doi:10.1016/0001-8708(86)90081-2.

BM02 C. Breuil and W. Messing, Torsion étale and crystalline cohomologies. Cohomologies p-adiques et applications arithmétiques, II, Astérisque (2002), no. 279, 81-124.

BO83 P. Berthelot and A. Ogus, F-isocrystals and de Rham cohomology. I, Invent. Math. 72 (1983), no. 2, 159-199; doi:10.1007/BF01389319.

CN17 P. Colmez and W. Niziol, Syntomic complexes and p-adic nearby cycles, Invent. Math. 208 (2017), no. 1, 1-108; doi:10.1007/s00222-016-0683-3.

CSS83 J.-L. Colliot-Thélène, J.-J. Sansuc, and C. Soulé, Torsion dans le groupe de Chow de codimension deux, Duke Math. J. 50 (1983), no. 3, 763-801; doi:10.1215/S0012-7094-83-05038-X.

FM87 J.-M. Fontaine and W. Messing, p-adic periods and p-adic étale cohomology, Current Trends in Arithmetical Algebraic Geometry (Arcata, Calif., 1985) (ed. K. Ribet), Contemp. Math., vol. 67 (Amer. Math. Soc., Providence, RI, 1987), 179-207; doi:10.1090/conm/067/902593.

Gei04 T. Geisser, Motivic cohomology over Dedekind rings, Math. Z. 248 (2004), no. 4, 773-794; doi:10.1007/s00209-004-0680-x.

GL00 T. Geisser and M. Levine, The K-theory of fields in characteristic p, Invent. Math. 139 (2000), no. 3, 459-493; doi:10.1007/s002220050014.

GL01_, The Bloch-Kato conjecture and a theorem of Suslin-Voevodsky, J. reine angew. Math. 530 (2001), 55-103; doi:10.1515/crll.2001.006.

Gro94 M. Gros, Régulateurs syntomiques et valeurs de fonctions L p-adiques. II, Invent. Math. 115 (1994), no. 1, 61-79; doi:10.1007/BF01231754.

HK94 O. Hyodo and K. Kato, Semi-stable reduction and crystalline cohomology with logarithmic poles, Astérisque (1994), no. 223, 221-268.

Ill79 L. Illusie, Complexe de de Rham-Witt et cohomologie cristalline, Ann. Sci. École Norm. Sup. (4) 12 (1979), no. 4, 501-661; doi:10.24033/asens. 1374. 


\section{ERTL AND W. NizioŁ}

Kat82 K. Kato, Galois cohomology of complete discrete valuation fields, Algebraic K-Theory, Part II (Oberwolfach, 1980), Lecture Notes in Math., vol. 967 (Springer, Berlin - New York, 1982), 215-238; doi:10.1007/BFb0061904.

Kat87_, On p-adic vanishing cycles (application of ideas of Fontaine-Messing), Algebraic Geometry (Sendai, 1985), Adv. Stud. Pure Math., vol. 10 (North-Holland, Amsterdam, 1987), 207251.

Kat89 L L L L L L L Fithmic structures of Fontaine-Illusie, Algebraic Analysis, Geometry, and Number Theory (Baltimore, MD, 1988) (Johns Hopkins Univ. Press, Baltimore, MD, 1989), 191-224.

Kat94 Semi-stable reduction and p-adic étale cohomology, Astérisque (1994), no. 223, 269-293.

KM92 K. Kato and W. Messing, Syntomic cohomology and p-adic étale cohomology, Tohoku Math. J. 44 (1992), no. 1, 1-9; doi:10.2748/tmj/1178227370.

Kur87 M. Kurihara, A note on p-adic étale cohomology, Proc. Japan Acad. Ser. A Math. Sci. 63 (1987), no. 7, 275-278; doi:10.3792/pjaa.63.275.

Lor02 P. Lorenzon, Logarithmic Hodge-Witt forms and Hyodo-Kato cohomology, J. Algebra 249 (2002), no. 2, 247-265; doi:10.1006/jabr.2001.8802.

Niz98 W. Nizioł, Crystalline conjecture via K-theory, Ann. Sci. École Norm. Sup. (4) 31 (1998), no. 5, 659-681; doi:10.1016/S0012-9593(98)80003-7.

Niz01_, Cohomology of crystalline smooth sheaves, Compos. Math. 129 (2001), no. 2, 123-147; doi:10.1023/A:1014577828651.

Niz06 , p-adic motivic cohomology in arithmetic, International Congress of Mathematicians, Vol. II (Eur. Math. Soc., Zürich, 2006), 459-472.

Niz08 Semistable conjecture via K-theory, Duke Math. J. 141 (2008), no. 1, 151-178; doi:10.1215/S0012-7094-08-14114-6.

Niz16_On syntomic regulators, I: constructions, 2016, arXiv:1607.04975.

NN16 J. Nekovář and W. Nizioł, Syntomic cohomology and p-adic regulators for varieties over p-adic fields (with appendices by Laurent Berger and Frédéric Déglise, Algebra Number Theory 10 (2016), no. 8, 1695-1790; doi:10.2140/ant.2016.10.1695.

Ogu90 A. Ogus, The convergent topos in characteristic $p$, in The Grothendieck Festschrift, Vol. III, Progr. Math., vol. 88 (Birkhäuser Boston, Boston, MA, 1990), 133-162; doi:10.1007/978-0-8176-4576-2_5.

Sat13 K. Sato, Cycle classes for p-adic étale Tate twists and the image of p-adic regulators, Doc. Math. 18 (2013), 177-247; https://www.emis.de/journals/DMJDMV/vol-18/07.html.

Shi02 A. Shiho, Crystalline fundamental groups. II. Log convergent cohomology and rigid cohomology, J. Math. Sci. Univ. Tokyo 9 (2002), no. 1, 1-163; http://www.ms.u-tokyo.ac.jp/journal/ abstract_e/jms090101_e.html.

SV00 A. Suslin and V. Voevodsky, Bloch-Kato conjecture and motivic cohomology with finite coefficients, The Arithmetic and Geometry of Algebraic Cycles (Banff, AB, 1998), NATO Sci. Ser. C Math. Phys. Sci., vol. 548 (Kluwer Acad. Publ., Dordrecht, 2000), 117-189; doi:10.1007/978-94-011-4098-0_5.

Tsu98 T. Tsuji, p-adic Hodge theory in the semi-stable reduction case, Proceedings of the International Congress of Mathematicians, Vol. II (Berlin, 1998), Doc. Math. (1998), Extra Vol. II, 207-216; https://www.emis.de/journals/DMJDMV/xvol-icm/03/Tsuji.MAN.html.

Tsu99 , p-adic étale cohomology and crystalline cohomology in the semi-stable reduction case, Invent. Math. 137 (1999), no. 2, 233-411; doi:10.1007/s002220050330.

Tsu00_, On p-adic nearby cycles of log smooth families, Bull. Soc. Math. France 128 (2000), no. 4, 529-575; doi:10.24033/bsmf. 2381 .

Wei09 C. Weibel, The norm residue isomorphism theorem, J. Topol. 2 (2009), no. 2, 346-372; doi:10.1112/jtopol/jtp013. 
Syntomic COHOMOLOGY AND p-ADIC MOTIVIC COHOMOLOGY

Veronika Ertl veronika.ertl@mathematik.uni-regensburg.de

Universität Regensburg, Fakultät für Mathematik Universitätsstrasse 31, 93053 Regensburg, Germany

Wiesława Nizioł wieslawa.niziol@ens-lyon.fr

CNRS, UMPA, École Normale Supérieure de Lyon, 46 allée d'Italie, 69007 Lyon, France 